\title{
Molecular gas in NUclei of GAlaxies (NUGA)
}

\section{The warped LINER NGC $3718^{\star}$}

\author{
M. Krips ${ }^{1,2}$, A. Eckart ${ }^{1}$, R. Neri ${ }^{2}$, J. U. Pott ${ }^{1,11}$, S. Leon ${ }^{3}$, F. Combes ${ }^{4}$, S. García-Burillo ${ }^{5}$, L. K. Hunt ${ }^{6}$, A. J. Baker ${ }^{7}$, \\ L. J. Tacconi ${ }^{8}$, P. Englmaier ${ }^{12}$, E. Schinnerer ${ }^{9}$, and F. Boone ${ }^{10}$ \\ 1 Universität zu Köln, I.Physikalisches Institut, Zülpicher Str. 77, 50937 Köln, Germany \\ e-mail: [krips; eckart]@ph1.uni-koeln.de \\ 2 Institut de Radio-Astronomie Millimétrique (IRAM), 300 rue de la Piscine, 38406 St. Martin-d'Hères, France \\ e-mail: neri@iram.fr \\ 3 Instituto de Astrofísica de Andalucía (CSIC), C/ Camino Bajo de Huétor 24, Apartado 3004, 18080 Granada, Spain \\ e-mail: stephane@iaa.es \\ ${ }^{4}$ Observatoire de Paris, LERMA, 61 Av. de l'Observatoire, 75014 Paris, France; e-mail: francoise.combes@obspm.fr \\ 5 Observatorio Astronómico Nacional (OAN)-Observatorio de Madrid, Alfonso XII 3, 28014 Madrid, Spain \\ e-mail: burillo@oan.es \\ ${ }^{6}$ Istituto di Radioastronomia/CNR, Sez. Firenze, Largo Enrico Fermi 5, 50125 Firenze, Italy \\ e-mail: hunt@arcetri.astro.it \\ 7 Jansky Fellow, National Radio Astronomy Observatory Department of Astronomy, University of Maryland, College Park, MD 20742-2421, \\ USA; e-mail: ajb@astro.umd.edu \\ 8 Max-Planck-Institut für extraterrestrische Physik, Postfach 1312, 85741 Garching, Germany; e-mail: linda@mpe.mpg.de \\ 9 Max-Planck-Institut für Astronomie, Königstuhl 17, 69117 Heidelberg, Germany; e-mail: schinner@mpia-hd.mpg.de \\ 10 Max Planck Institut für Radioastronomie, Auf dem Hügel 69, 53121 Bonn, Germany \\ e-mail: fboone@mpifr-bonn.mpg.de \\ 11 ESO, Karl-Schwarzschild-Str. 2, 85748 Garching, Germany; e-mail: jpott@eso.org \\ 12 Astronomisches Institut, Universität Basel, Venusstr. 7, 4102 Binningen, Switzerland; e-mail: ppe@astro.unibas.ch
}

Received 26 July 2004 / 15 July 2005

\begin{abstract}
We present the first interferometric observations of $\mathrm{CO}(1-0)$ and $\mathrm{CO}(2-1)$ line emission from the warped LINER NGC 3718 , obtained with the IRAM Plateau de Bure Interferometer (PdBI). This L1.9 galaxy has a prominent dust lane and on kiloparsec scales, a strongly warped atomic and molecular gas disk. The molecular gas is closely associated with the dust lane across the nucleus and its kinematic center is consistent with the millimeter continuum AGN. A comparison of our interferometric mosaic data, which fully cover the $\sim 9 \mathrm{kpc}$ warped disk, with a previously obtained IRAM $30 \mathrm{~m}$ single dish $\mathrm{CO}(1-0)$ map shows that the molecular gas distribution in the disk is heavily resolved by the PdBI map. On the nucleus the interferometric maps alone contain less than one half of the single dish line flux, and the overall mosaic accounts for about a tenth of the total molecular gas mass of $\sim 2.4 \times 10^{8} \mathcal{M}_{\odot}$. After applying a short-spacing correction with the IRAM $30 \mathrm{~m}$ data to recover the missing extended flux, we find in total six main source components within the dust lane: one associated with the nucleus, four symmetrically positioned on either side at galactocentric distances of about $1.3 \mathrm{kpc}$ and $4.0 \mathrm{kpc}$ from the center, and a sixth on the western side at $\sim 3 \mathrm{kpc}$ with only a very weak eastern counterpart. In the framework of a kinematic model using tilted rings, we interpret the five symmetric source components as locations of strong orbital crowding. We further find indications that the warp appears not only on kpc scales, but continues down to $\sim 250 \mathrm{pc}$. Besides the sixth feature on the western side, the lower flux (a factor of $\sim 2$ ) of the eastern components compared to the western ones indicates an intrinsic large scale asymmetry in NGC 3718 that cannot be explained by the warp. Indications for a small scale asymmetry are also seen in the central $600 \mathrm{pc}$. These asymmetries might be evidence for a tidal interaction with a companion galaxy (large scales) and gas accretion onto the nucleus (small scales). Our study of NGC 3718 is part of the NUclei of GAlaxies (NUGA) project that aims at investigating the different processes of gas accretion onto Active Galactic Nuclei (AGN).
\end{abstract}

Key words. galaxies: individual: NGC 3718 - galaxies: active - Galaxy: kinematics and dynamics

\footnotetext{
* Based on observations carried out with the IRAM Plateau de Bure Interferometer. IRAM is supported by INSU/CNRS (France), MPG (Germany) and IGN (Spain).
} 


\section{Introduction}

Knowledge of the distribution and kinematics of the circumnuclear molecular gas in active galaxies is essential for understanding the fueling of the central engine and the role of gas and dust in obscuring the active nucleus (AGN). Although we have begun to understand the factors that influence nuclear activity, a wealth of unanswered questions still remain. In contrast to large scales $(\geq 3 \mathrm{kpc}$ ) where dynamical perturbations like galaxy collisions, mergers, or mass accretion (Heckman et al. 1986) as well as bars, spirals and their gravity torques (Combes 1988) are responsible for the infall of gas, the processes responsible for removing angular momentum at small scales (sub-kpc) are not very well understood. Various scenarios invoking nested bars (e.g., Shlosman et al. 1993), spirals (e.g., Martini \& Pogge 1999), warped nuclear disks (Schinnerer et al. 2000a,b), and lopsidedness or $m=1$ instabilities (Kormendy \& Bender 1999; García-Burillo et al. 2000) have been proposed. Millimeter interferometry allows qualitative studies of molecular gas emission in these galaxies with arcsecond spatial resolution (and even below), high spectral resolution ( $\sim$ a few $\mathrm{km} \mathrm{s}^{-1}$ per channel) and high sensitivity (detectability thresholds $\sigma_{\text {gas }} \sim$ a few tens $\mathcal{M}_{\odot} \mathrm{pc}^{-2}$ ). The NUclei of GAlaxies (NUGA) project (García-Burillo et al. 2003a,b) aims at establishing a high-resolution and high-sensitivity $\mathrm{CO}$ survey of 12 nearby AGN covering the full sequence of activity types including Seyfert 1 and Seyfert 2 galaxies as well as Low Ionization Nuclear Emission-line Region (LINER) and transition objects. The $\mathrm{CO}$ observations are conducted with the IRAM Plateau de Bure Interferometer (PdBI: Guilloteau et al. 1992), which currently provides the highest sensitivity and angular resolution for the study of the distribution and dynamics of molecular gas in the nuclei of these galaxies. Besides case by case analyses and simulations of each object (García-Burillo et al. 2003b; Combes et al. 2004), the collected data will also be used for a first-order approach to a statistical study of how gas flows into nuclei and the different mechanisms that account for further accretion inward.

One of the active galaxies belonging to the NUGA survey is NGC 3718 (Fig. 1), a peculiar, elliptical galaxy at a distance of $13 \mathrm{Mpc}$ (Pott et al. 2004; Schwarz 1985). Together with its companion NGC 3729 it belongs to the Ursa Major group. It still remains unclear if and to what extent a gravitational interaction is taking place between these two galaxies. A large warped dust lane runs across the entire stellar bulge of NGC 3718; it has a width of $<2$ " at the center and diverges into several smooth filaments across the bulge ( $1^{\prime \prime}$ corresponds to $\sim 64 \mathrm{pc}$ for NGC 3718 ). At a radius of about $1.5^{\prime}$ from the center, the dust lane bends by almost 90 degrees towards the north and south. This warp signature is also observed in the cold gas distribution, with the $\mathrm{H}$ I line emission tracing it out to a radius of more than $6^{\prime}(24 \mathrm{kpc})$ and $\mathrm{CO}$ line emission tracing it towards the center $\left(\sim 20^{\prime \prime}\right.$ : Pott et al. 2004). The outer parts of the warp were kinematically modeled by Schwarz (1985), while the inner parts down to $\sim 20^{\prime \prime \prime}$ were modelled by Pott et al. (2004).

NGC 3718 is classified as a LINER galaxy of type 1.9 (Ho et al. 1997). Weak, broad $\mathrm{H} \alpha$ emission with $F W H M$

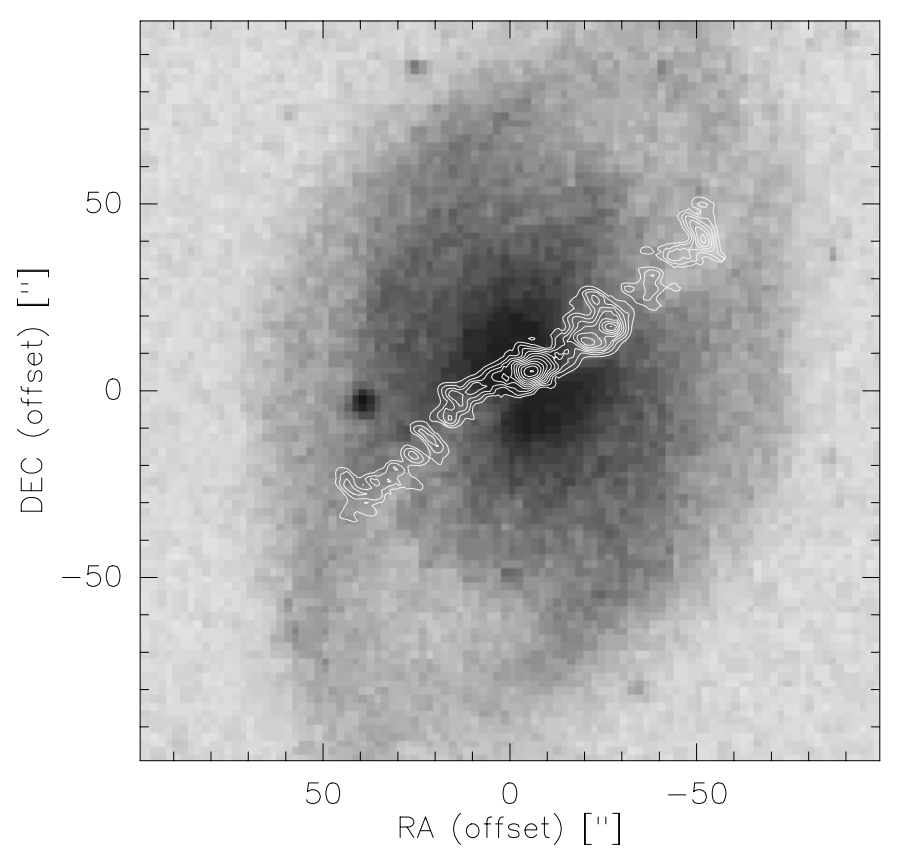

Fig. 1. Optical image (taken from the DSS survey) superimposed with the integrated $\mathrm{CO}(1-0)$ contours (white) obtained at the PdBI, with added short spacings from the IRAM $30 \mathrm{~m}$ telescope (see Sects. 4.2 and 4.3). Contour levels as in Fig. 14.

$2350 \mathrm{~km} \mathrm{~s}^{-1}$ is emitted from the nucleus as well as strong [O I] $\lambda=6300 \AA$ with $F W H M 570 \mathrm{~km} \mathrm{~s}^{-1}$ indicative of a hidden AGN (Filippenko et al. 1985; Ho et al. 1997). So far no ultraviolet emission has been detected towards NGC 3718 (Barth et al. 1998), probably due to obscuration of the nucleus by dust, whereas a radio source has been confirmed at the position of the nucleus (this work; Burke \& Miley 1973; Nagar et al. 2002).

In this paper we analyze single field and mosaic data obtained with the PdBI at an angular resolution of $\sim 4^{\prime \prime}$. Section 2 describes the observations. In Sect. 3 we present the results from the observations. A kinematic model is outlined in Sect. 4. We finish with a discussion.

\section{Observations}

NGC 3718 was observed with the IRAM $30 \mathrm{~m}$ and the IRAM PdBI. The IRAM $30 \mathrm{~m}$ observations are already described in detail by Pott et al. (2004) and will thus here only be discussed in the context of the short-spacing correction and resolution effects. The IRAM PdBI observations were carried out in two different modes: central pointing (one single field on the center) and mosaic (seven fields along the dust lane).

\subsection{Central pointing}

During winter 2000/2001, we mapped the $\mathrm{CO}(1-0)$ and $\mathrm{CO}(2-$ 1) lines in a single field centered at the radio position of the $\mathrm{AGN}$ in NGC 3718 , i.e., $\alpha_{2000}=11^{\mathrm{h}} 32^{\mathrm{m}} 34.840^{\mathrm{s}}$ and $\delta_{2000}=$ $53^{\circ} 04^{\prime} 04.90^{\prime \prime}$ (see Sect. 3.1.1). The PdBI was at this time deployed in the $\mathrm{CD}$ set of configurations with 5 antennae. The bandpass calibrator was 3C 273, while the phase and amplitude calibration were performed on $1150+497$. Fluxes have been 
calibrated relative to CRL618 and MWC349. The frequencies were centered on the redshifted ${ }^{12} \mathrm{CO}(1-0)$ line in the USB at $3 \mathrm{~mm}$ and on the redshifted ${ }^{12} \mathrm{CO}(2-1)$ line in the LSB at $1 \mathrm{~mm}$. For each line, the total bandwidth was $580 \mathrm{MHz}$ and the spectral resolution $1.25 \mathrm{MHz}$. The integration time for the central pointing amounts to $\sim 8 \mathrm{~h}$ on source. This gives a point source sensitivity of about $\sim 7 \mathrm{mJy}(\sim 16 \mathrm{mJy})$ in channels of $1.25 \mathrm{MHz}$ width at $3 \mathrm{~mm}(1 \mathrm{~mm})$. The precipitable water vapor ranged between 4 and $10 \mathrm{~mm}$ (i.e., opacities of $\sim 0.2-0.3$ ) resulting in system temperatures of approximately $200-300 \mathrm{~K}$ on average. We used the GILDAS software packages to reduce and map the data (Guilloteau \& Lucas 2000). The synthesized beams are $4.7^{\prime \prime} \times 3.5^{\prime \prime}$ at $41^{\circ}$ at $3 \mathrm{~mm}$ and $2.6^{\prime \prime} \times 2.0^{\prime \prime}$ at $45^{\circ}$ at $1 \mathrm{~mm}$.

\subsection{Mosaic}

During winter 2001/2002, a mosaic of NGC 3718 was obtained with the PdBI. Seven mosaic fields of the $\mathrm{CO}(1-0)$ emission were repeatedly observed with six antennae or a subset in $\mathrm{C}$ and $\mathrm{D}$ configurations. These sample the molecular gas emission along the dust lane with $\sim 12 \mathrm{kpc}$ extent that was previously mapped with the IRAM $30 \mathrm{~m}$ by Pott et al. (2004) (Fig. 2; see also Hartwich et al. 2002 and Krips et al. 2003). The central field of the mosaic was set to the same position specified in Sect. 2.1, and the separation between the pointings was $21^{\prime \prime}$, corresponding to half the primary beam size. The bandpass was calibrated on 3C 84, 3C 273, or $0923+392$ with an accuracy of about $5 \%$. The latter source and $1150+497$ were used as phase and amplitude calibrator, observed every $20 \mathrm{~min}$. To derive the absolute fluxes of the calibrators and the target source, values for each observing epoch were bootstrapped via a standard IRAM flux monitoring program, yielding an accuracy of the flux calibrations of $\sim 10 \%$ at $3 \mathrm{~mm}$ and $\sim 20 \%$ at $1 \mathrm{~mm}$.

The spectral resolution was set to $1.25 \mathrm{MHz}$ at $114.89 \mathrm{GHz}$, giving a contiguous bandwidth of $580 \mathrm{MHz}$. The integration time per field amounted to $\sim 2.4 \mathrm{~h}$. The resulting pointsource sensitivity is $\sim 17 \mathrm{mJy}$ for a bandwidth of $1.25 \mathrm{MHz}$ and 5 antennae at $3 \mathrm{~mm}$. The precipitable water vapor ranged between 4 and $10 \mathrm{~mm}$ (i.e., opacities of $\sim 0.2-0.3$ ) resulting in system temperatures of approximately $200-300 \mathrm{~K}$ on average. Even if the $\mathrm{CO}(2-1)$ line had been simultaneously observed in mosaic mode, the mosaic fields would only have been usable as single fields and not as complete mosaic since the separations of the mosaic fields are too large at $1 \mathrm{~mm}$ where the primary beam size is $\sim 21^{\prime \prime}$. Also, the sensitivity is too poor in most of the fields, resulting in no detection of the $\mathrm{CO}(2-1)$ line except in the central field.

\section{The data}

\subsection{Central emission}

\subsubsection{Continuum}

Before combining the central pointing observation (singlefield) with the new central field from the mosaic observations, the continuum for both data sets at $1 \mathrm{~mm}$ and $3 \mathrm{~mm}$ has been independently determined from the line free channels. In both

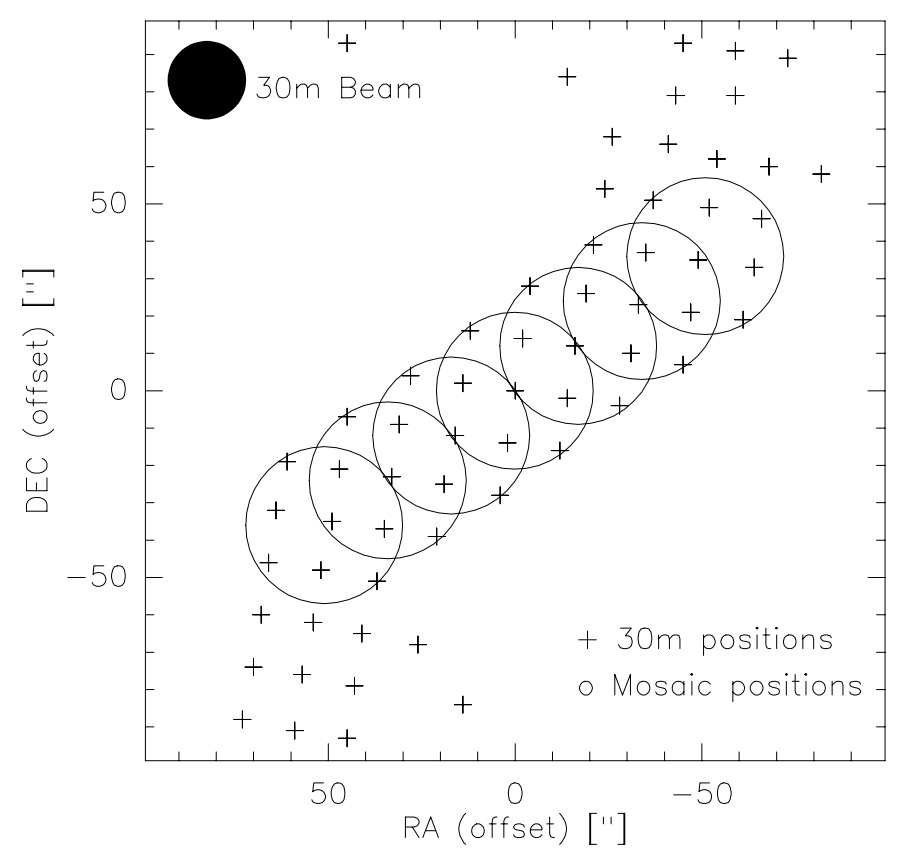

Fig. 2. Positions of the IRAM $30 \mathrm{~m}$ observations are plotted with black crosses, and mosaic fields of the PdBI observations are shown as circles indicating the $42^{\prime \prime}$ primary beam size for each observing field. The $30 \mathrm{~m}$ beamsize is shown in the upper left.

data sets at $3 \mathrm{~mm}$, i.e. single field and central mosaic field, we detect faint continuum emission in the central region of NGC 3718. At $1 \mathrm{~mm}$, only the single-field data set reveals continuum emission (see Fig. 3); we do not find any emission in the central mosaic field probably due to sensitivity limitations for the $1 \mathrm{~mm}$ mosaic data. At $3 \mathrm{~mm}$ as well as at $1 \mathrm{~mm}$, the continuum emission is point-like relative to the respective beams (compare Table 1). The continuum declines by $\sim 30 \%$ between the two observing epochs at $3 \mathrm{~mm}$ (see Table 1). Assuming an upper limit of $3 \sigma=7.5 \mathrm{mJy}^{-1}$ beam $^{-1}$ at $1 \mathrm{~mm}$ for the continuum of the central field from the mosaic, we obtain a decline of $\geq 20 \%$ relative to the single field data (flux $\sim 10 \mathrm{mJy} \mathrm{beam}^{-1}$ ) consistent with the $3 \mathrm{~mm}$ data. As the atmospheric conditions were quite similar (similar amounts of water vapor) between both data sets, we can mostly exclude atmospheric absorption which could cause an artificial drop of the flux between the data. The phase and amplitude calibrators were never separated from NGC 3718 by more than a few degrees in elevation. The $\sim 10 \%$ uncertainty in $3 \mathrm{~mm}$ flux calibration cannot explain all of the observed variability, although this is not the case at $1 \mathrm{~mm}$. We do not find any support for a variable continuum source at other wavelengths in recent EVN and MERLIN measurements at $18 \mathrm{~cm}$ and $6 \mathrm{~cm}$ (Krips et al. in prep., epochs 2001 to 2004) or other $\mathrm{cm}$ observations (at $2 \mathrm{~cm}$ and $3.6 \mathrm{~cm}$; e.g., Nagar et al. 2002). In conclusion, the detected mm-variability might be an intrinsic property of NGC 3718 but has to be further investigated.

The derived positions are consistent between the two observing epochs as well as between $1 \mathrm{~mm}$ and $3 \mathrm{~mm}$. They also agree with the positions of the nucleus obtained at $\mathrm{cm}$ and optical wavelengths within the errors. Accounting for a weak variability, a comparison between our mm fluxes and $\mathrm{cm}$ fluxes 

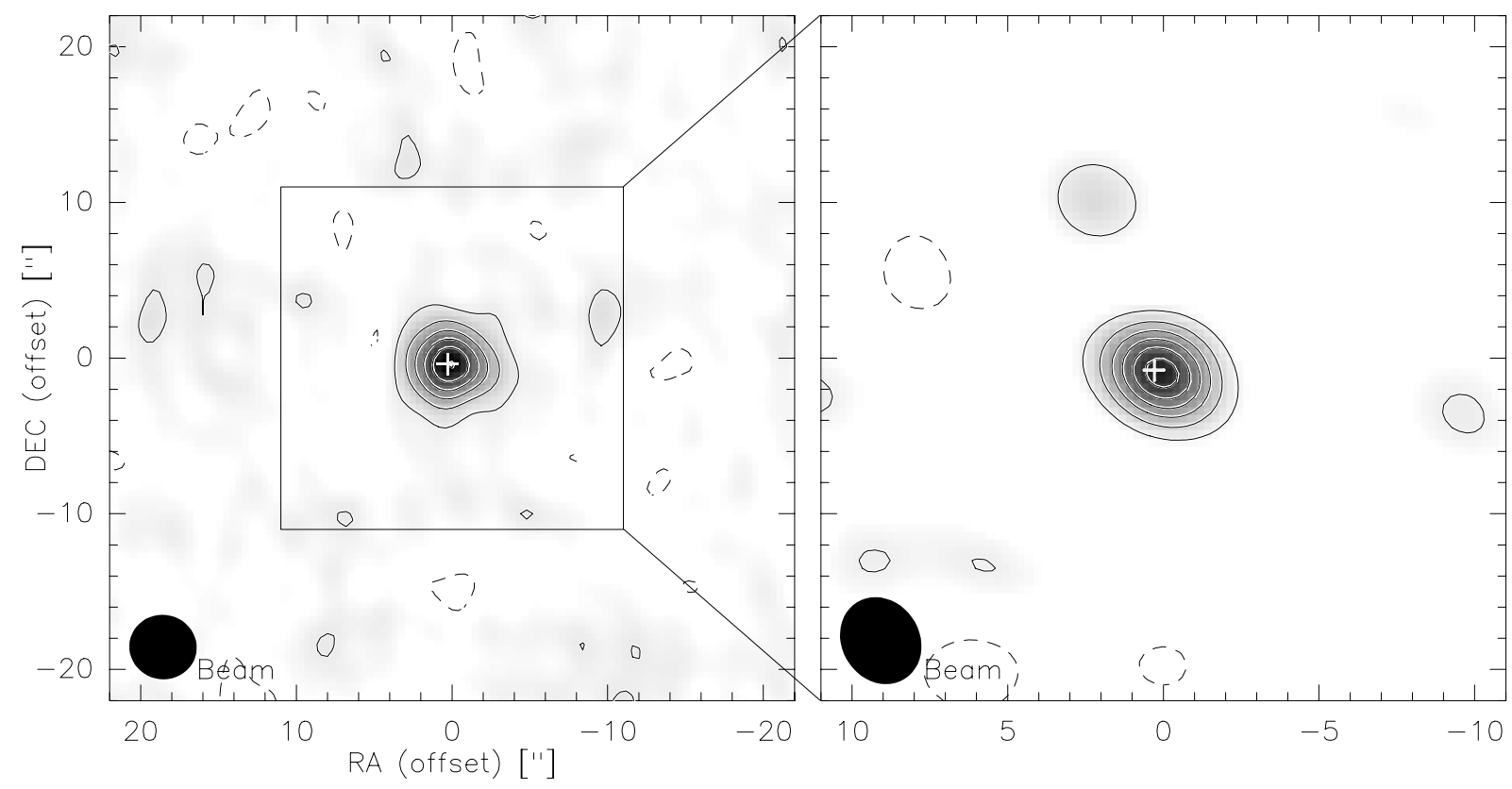

Fig. 3. Continuum emission of the single field observations at $3 \mathrm{~mm}$ (left $)$ and $1 \mathrm{~mm}$ (right). The white cross indicates the position at $18 \mathrm{~cm}$ with EVN. Contour levels are from $2 \sigma(2 \sigma)=1.0(1.6) \mathrm{mJy} \mathrm{beam}^{-1}$ to $9(9.6) \mathrm{mJy} \mathrm{beam}^{-1}$ in steps of $4 \sigma(2 \sigma)$ at $3 \mathrm{~mm}(1 \mathrm{~mm})$. Beam sizes are indicated at lower left.

Table 1. Interferometric positions and fluxes of the continuum emission at $1 \mathrm{~mm}$ and $3 \mathrm{~mm}$ from the single field data set (2000/01) and the central field of the mosaic observations from 2001/02 (right column). The difference in the $3 \mathrm{~mm}$ flux densities between the first epoch and the second is discussed in the text (Sect. 3.1.1).

\begin{tabular}{|c|c|c|c|}
\hline & \multicolumn{2}{|c|}{ - 2000/01 } & \multirow{3}{*}{$\begin{array}{c}-2001 / 02- \\
\text { cont@ } \\
3 \mathrm{~mm}\end{array}$} \\
\hline & cont@ & cont@ & \\
\hline & $3 \mathrm{~mm}$ & $1 \mathrm{~mm}$ & \\
\hline RA & $11: 32: 34.852$ & $11: 32: 34.878$ & $11: 32: 34.83$ \\
\hline (hhmmss) & \pm 0.003 & \pm 0.005 & \pm 0.01 \\
\hline Dec & 53:04:04.51 & 53:04:04.3 & $53: 04: 04.2$ \\
\hline$\left({ }^{\circ}:^{\prime}:^{\prime \prime}\right)$ & \pm 0.06 & \pm 0.1 & \pm 0.1 \\
\hline $\begin{array}{l}\text { peak flux } \\
\left(\mathrm{mJy} \text { beam }^{-1}\right)\end{array}$ & $9.4 \pm 0.7$ & $10 \pm 2$ & $7 \pm 1$ \\
\hline
\end{tabular}

observed with the VLA in 1997 (Becker et al. 1995), 1998 (Nagar et al. 2001), and 1999 (Nagar et al. 2002) indicates a spectral index of $-0.01 \pm 0.1$ depending on which of our two observing epochs is used. This corresponds to a flat to inverted spectrum for the central source in NGC 3718, like those found for many other low-luminosity active nuclei in Seyfert galaxies and LINERs (Wrobel \& Heeschen 1991; Slee et al. 1994; Nagar et al. 2000). The flat radio spectra exclude emission from optically thin synchrotron emission, as is often seen from extended radio jets, since this produces steep radio spectra. Thus, synchrotron self-absorption or advection dominated accretion flows (ADAFs: Narayan et al. 1998; Quataert 2001) have to be considered. A compact jet in combination with an ADAF (Falcke 1996; Falcke \& Marloff 2000; Falcke 2001) also cannot be excluded. If a longitudinal pressure gradient were to cause the jet to accelerate rapidly, the integrated radio emission of the compact core jet would also have a slightly inverted radio spectrum.

\subsubsection{Line}

After subtracting the continuum emission in the $u v$ plane from the (continuum+line) channels, $\mathrm{CO}(1-0)$ and $\mathrm{CO}(2-1)$ line emission is detected in the central $\sim 10^{\prime \prime}$ ( $\equiv 1 \mathrm{kpc}$ ) of NGC 3718. The combined central maps are shown in Fig. 4. In both lines the emission is slightly extended. The positions of the integrated $\mathrm{CO}(1-0)$ and $\mathrm{CO}(2-1)$ emission are similar to the nuclear position derived from the radio. Also, the isovelocity diagram in Fig. 7 shows that the dynamical center of the $\mathrm{CO}$ emission lies on the radio nucleus. A comparison of the flux observed with the PdBI and the IRAM $30 \mathrm{~m}$ single dish reveals that the interferometer recovers less than one half of the peak line flux seen with the $30 \mathrm{~m}$ telescope (see Fig. 6). This indicates that the emission in NGC 3718 must be much more extended and diffuse than is apparent in the integrated map of Fig. 4. Assuming a standard $M_{\text {gas }}\left(=M\left(\mathrm{H}_{2}+\mathrm{He}\right)=\right.$ $\left.1.36 \times M\left(\mathrm{H}_{2}\right)\right)$ to $L_{\mathrm{CO}(1-0)}^{\prime}$ ratio of $\sim 5 \mathcal{M}_{\odot}\left(\mathrm{Kkm} \mathrm{s}^{-1} \mathrm{pc}^{2}\right)^{-1}$ (Solomon \& Barrett 1991), the central molecular gas mass $M_{\text {gas }}$ amounts to $\sim 1 \times 10^{7} \mathcal{M}_{\odot}$ in the PdBI map, in contrast to $3 \times 10^{7} \mathcal{M}_{\odot}$ in the $30 \mathrm{~m}$ map (Pott et al. 2004). Downes et al. (1993) emphasize, however, that the standard galactic conversion factor between gas mass and $\mathrm{CO}$ luminosity might fail for the centers of galaxies where the molecular gas is bound by the total gravitational potential of the galaxy rather than by selfgravity. In such a case, the conversion factor would be up to 5 times lower than the standard galactic one, resulting in gas masses of $\sim 0.2 \times 10^{7} \mathcal{M}_{\odot}$ for the PdBI map and $0.6 \times 10^{7} \mathcal{M}_{\odot}$ for the $30 \mathrm{~m}$ map.

By comparing the distributions of the central $\mathrm{CO}(1-0)$ and $\mathrm{CO}(2-1)$ line emission, we find that the $\mathrm{CO}(1-0)$ emission shows an elongation towards the south, almost perpendicular to the beam. We estimate the integrated line ratio $R_{21}=$ $\mathrm{CO}(2-1) / \mathrm{CO}(1-0)$ to $\sim 0.6$ indicating subthermal excitation 


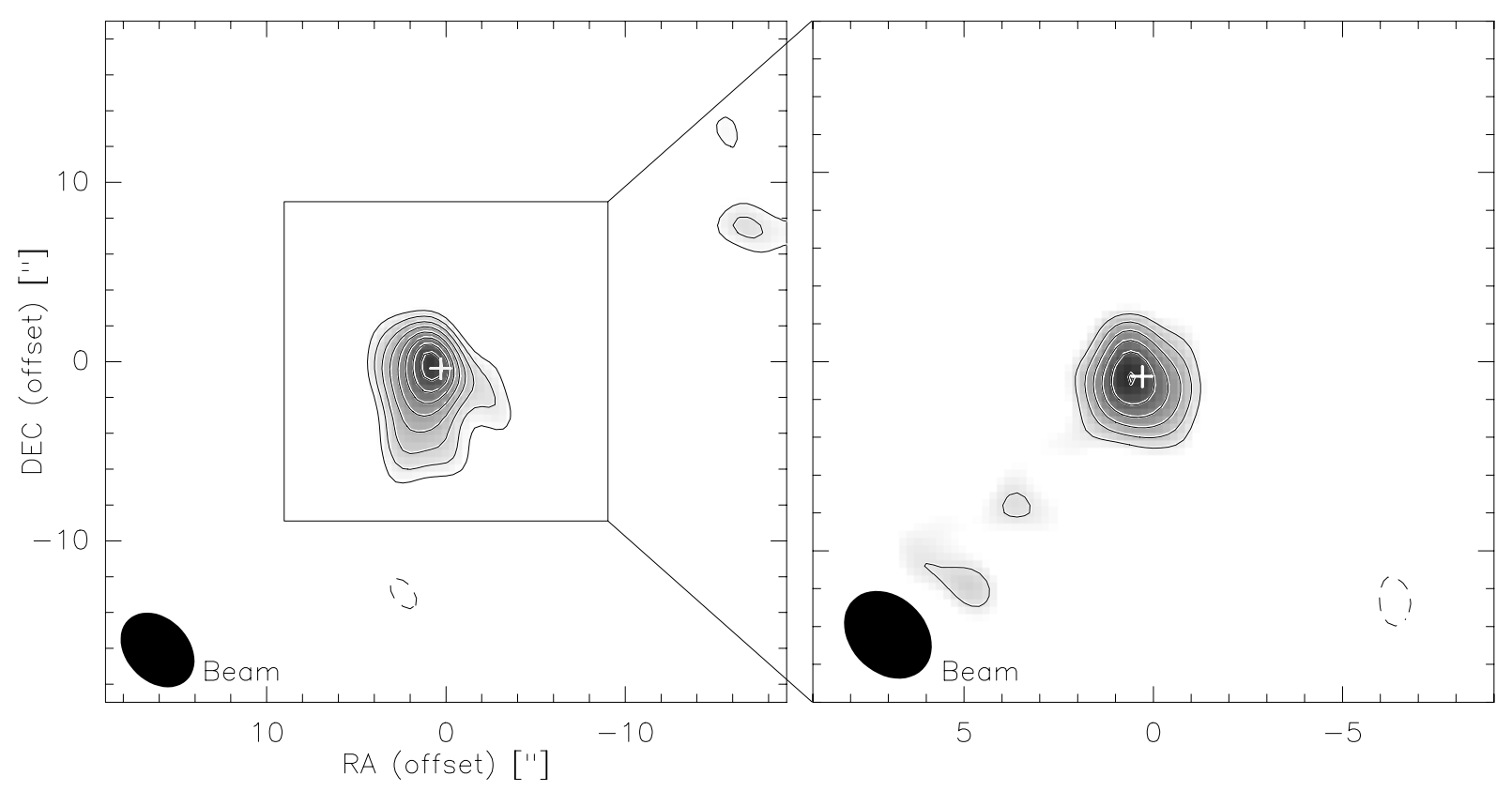

Fig. 4. PdBI central maps of the velocity integrated $\mathrm{CO}(1-0)$ (left) and $\mathrm{CO}(2-1)$ (right) emission in NGC 3718 (from velocities -300 to $\sim+300 \mathrm{~km} \mathrm{~s}^{-1}$ ). Contour levels are from $3 \sigma(3 \sigma)=1.5(2.7)$ to $5(7.2) \mathrm{Jy} \mathrm{beam}^{-1} \mathrm{~km} \mathrm{~s}^{-1}$ in steps of $1 \sigma(1 \sigma)$ for $\operatorname{CO}(1-0)(\operatorname{CO}(2-1))$. The white cross indicates the position at $18 \mathrm{~cm}$ with the European VLBI Network (EVN; Krips et al. 2005, in prep.).

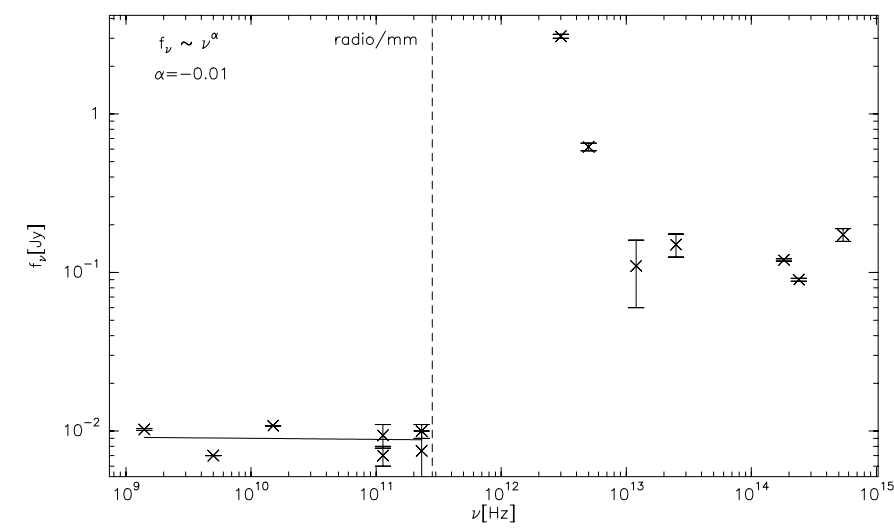

Fig. 5. The Spectral Energy Distribution (SED) of NGC 3718. IR and optical fluxes with their errors are shown for completeness (taken from NED). The $\mathrm{cm}$ fluxes were observed with the VLA (FIRST: Becker et al. 1995; Nagar et al. 2001, 2002) and thus have an angular resolution comparable to the PdBI maps. The mm fluxes for both observing epochs are plotted (upper limit at $1 \mathrm{~mm}$ ). The solid line indicates a fit $\left(f_{v} \propto v^{\alpha}\right)$ with $\alpha=-0.01 \pm 0.1$.

conditions and cold gas. To obtain this value, we have first truncated the inner $u v$ region of the $\mathrm{CO}(1-0)$ data and the outer $u v$ region of the $\mathrm{CO}(2-1)$ data to get comparable $u v$ coverages and resolutions for the two transitions, integrated both lines over the same velocity range of $-300 \mathrm{~km} \mathrm{~s}^{-1}$ to $+300 \mathrm{~km} \mathrm{~s}^{-1}$ and then transformed the flux (Jansky) into temperature (Kelvin). However, due to the limited signal-to-noise and the difference of the weighting factors between both transitions, the reliability of this estimate is still questionable and thus the value of $\sim 0.75$ derived in the center by Pott et al. (2004) might be a better indicator for the physical conditions in NGC 3718.
Figure 7 shows the zero order moment maps of both transitions overlaid with contours from the first order moment maps and the position velocity cuts taken along the kinematic major axis. The $\mathrm{CO}(2-1)$ maps are quite noisy but are plotted for consistency and comparison purposes. The position velocity diagram of the $\mathrm{CO}(1-0)$ line emission reveals a quite extended $\left(\sim 8^{\prime \prime}\right)$ velocity gradient between $\pm 150 \mathrm{~km} \mathrm{~s}^{-1}$. Two further features appear at very high velocities $\left(\sim \pm 220 \mathrm{~km} \mathrm{~s}^{-1}\right)$ with a somewhat steeper gradient $\left(\sim \pm 2^{\prime \prime}\right)$. Consistent with Pott et al. (2004), the global velocity range of the inner $\sim 15^{\prime \prime}$ amounts to almost $\pm 220 \mathrm{~km} \mathrm{~s}^{-1}$. However, this "central" feature in the position velocity diagram extends in Pott et al. (2004) out to a radius of 15-20", while in our interferometric data it stops at a radius of $\lesssim 10^{\prime \prime}$. This rapidly rotating nuclear component will be discussed in Sect. 4.3. The line emission shows a strong asymmetry in the position velocity diagram with only little emission towards the blueshifted velocities. Section 3.4 will show that this asymmetry cannot be totally traced back to missing short spacings.

\subsection{Overall emission}

\subsection{PdBI-only data}

Outside the central mosaic field, there is no evidence of continuum emission in our data. The $30 \mathrm{~m}$ map of Pott et al. (2004) has already revealed the existence of two additional maxima in the $\mathrm{CO}(1-0)$ emission which are offset by $\sim 30-40^{\prime \prime}$ from the nucleus. We find further maxima in the new PdBI mosaic map which are located farther out at $\sim \pm 60^{\prime \prime}$ (compare Figs. 10 and 14). In Fig. 10 we have labeled the eastern components SE1 and SE2, the western components NW1 and NW2, and the central feature $\mathrm{C}$. There are also indications for further 


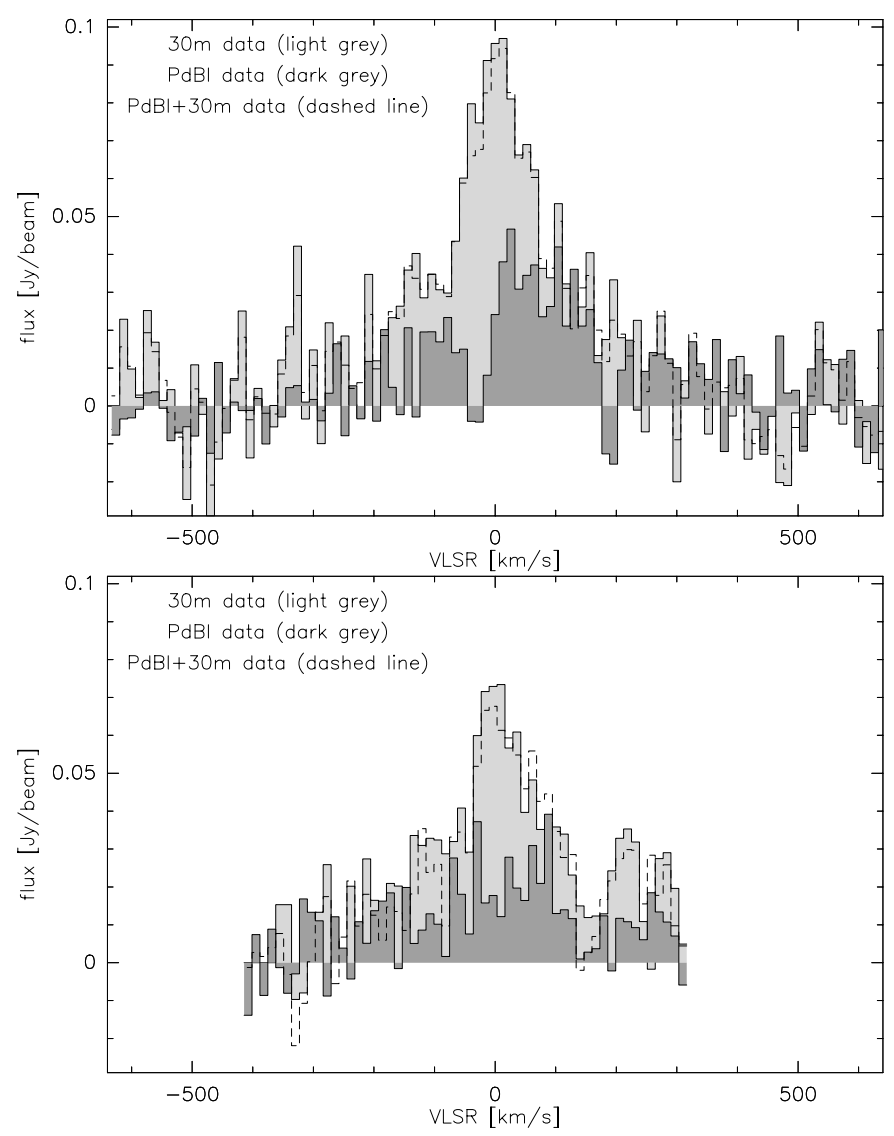

Fig. 6. $\mathrm{CO}(1-0)$ (upper panel) and $\mathrm{CO}(2-1)$ (upper panel) spectra of the central emission in NGC 3718 observed with the PdBI (dark grey; integrated over the emission area), the IRAM $30 \mathrm{~m}$ telescope (light grey; Pott et al. 2004). The short-spacing corrected spectrum is also shown (dashed line; integrated over the emission area). Spectra are continuum subtracted. The rms noise level is here $\sim 4 \mathrm{mJy}(\sim 6 \mathrm{mJy})$ for the PdBI data for $\mathrm{CO}(1-0)(\mathrm{CO}(2-1))\left(\Delta v=13 \mathrm{~km} \mathrm{~s}^{-1}\right.$ for both).

maxima between NW1 and NW2 and also between SE1 and SE2 but they still lack enough sensitivity $(\leq 2 \sigma)$ in the PdBIonly map to be further substantiated at this point. The brightest line in the spectra turns out to be NW2 and C, whereas the other three features appear to be rather weak with a signal-to-noise ratio at line peak of $\sim 4$ (Fig. 10). However, NW2 appears to be quite weak in the integrated map which might be traced back to its weak line width of only $\sim 50 \mathrm{~km} \mathrm{~s}^{-1}$. The integrated map was produced by averaging the channel maps over a total velocity width of $\sim 600 \mathrm{~km} \mathrm{~s}^{-1}$. A comparison between the global fluxes derived from the $30 \mathrm{~m}$ and the PdBI data again shows that a large part of the - diffuse, extended - flux (at least $\sim 70 \%$ of the total flux) must be resolved by the interferometer due to missing short spacings. Adding up all the PdBI spectra leads to a total molecular gas mass of $M_{\text {gas }} \sim 2.5 \times 10^{7} \mathcal{M}_{\odot}$. This is a tenth ${ }^{1}$ of the total molecular gas mass derived from the $30 \mathrm{~m}$ spectra of $\sim 2.4 \times 10^{8} \mathcal{M}_{\odot}$ (Pott et al. 2004).

\footnotetext{
${ }^{1}$ Besides the decreased flux measured with the PdBI also the line width is lower by $\lesssim 20 \%$ with respect to the $30 \mathrm{~m}$ data.
}

Table 2. Positions and fluxes for $\mathrm{CO}(1-0)$ components in the $\mathrm{PdBI}+30 \mathrm{~m}$ map. Offsets are with respect to $\alpha_{2000}=11 \mathrm{~h} 32 \mathrm{~m} 34.8$ and $\delta_{2000}=53^{\circ} 04^{\prime} 04.9^{\prime \prime}$.

\begin{tabular}{crrc}
\hline \hline Component & $\begin{array}{c}\text { RA (offset) } \\
{\left[{ }^{\prime \prime}\right]}\end{array}$ & $\begin{array}{c}\text { Dec. (offset) } \\
{\left[{ }^{\prime \prime}\right]}\end{array}$ & $\begin{array}{c}\text { Flux } \\
\mathrm{Jy} \mathrm{km} \mathrm{s}^{-1}\end{array}$ \\
\hline SE2 & $+52 \pm 3$ & $-34 \pm 3$ & 1.2 \\
SE1 & $+20 \pm 3$ & $-8 \pm 3$ & 1.3 \\
C & $0 \pm 1$ & $0 \pm 1$ & 3.4 \\
NW1 & $-19 \pm 1$ & $+9 \pm 1$ & 2.9 \\
NW2 & $-49 \pm 1$ & $+36 \pm 1$ & 2.7 \\
\hline
\end{tabular}

\subsection{PdBI+30 m data: short spacing correction}

To restore missing extended and diffuse emission, we combined the $\mathrm{CO}(1-0)$ data from the IRAM $30 \mathrm{~m}$ telescope with our interferometric maps. We varied the weights attached to the $30 \mathrm{~m}$ and PdBI data to find the best compromise between good angular resolution and almost complete restoration of the missing extended flux. In the end, a factor of 4 was applied to the weights of the $30 \mathrm{~m}$ data, leading to the recovery of $\sim 90 \%$ of the missing flux (see below) at a still reasonable angular resolution of $7.8 \times 4.5^{\prime \prime} @ 20^{\circ}$. A higher weighting factor would have increased the image restoration to over $90 \%$ but at the same time also decreased the resolution to $\sim 10^{\prime \prime}$, while a lower weighting factor would have had a higher angular resolution but a worse image restoration due to a lower signal-to-noise ratio. The combined maps of $30 \mathrm{~m}$ and PdBI data have finally been cleaned using standard program packages provided by the GILDAS software for mosaic data. The results are presented in Figs. 8 and 11 for the single field and mosaic maps. In Fig. 9, we compare the position-velocity diagrams along the axis used in Fig. 7 for the PdBI-only data and the PdBI+30 $\mathrm{m}$ data. The asymmetry discussed in Sect. 3.1.2 is still present even if slightly weakened and hence cannot be totally traced back to missing short spacings. This indicates that the asymmetry seems to be an intrinsic property of NGC 3718. A first comparison between the PdBI-only and the PdBI $+30 \mathrm{~m}$ maps shows in the mosaic maps that the short spacing correction strengthens the inner components (NW1, C and SE1) that appear to contain most of the extended and diffuse emission. If we now derive the total molecular gas mass, we obtain $\sim 2.1 \times 10^{8} \mathcal{M}_{\odot}$, which is $\sim 90 \%$ of the value found for the $30 \mathrm{~m}$-only data. Besides the five main emission peaks seen in Fig. 10, further emission between NW1 and NW2 and SE1 and SE2, as already indicated in the PdBI-only map (Fig. 10), is substantiated in the PdBI $+30 \mathrm{~m}$ map. Their gas masses are estimated to $\sim 0.2 \times 10^{8} \mathcal{M}_{\odot}$, respectively. Globally, the western part of the $\mathrm{CO}(1-0)$ emission appears to contain more gas $\left(M_{\text {gas }} \simeq 10^{8} \mathcal{M}_{\odot}\right.$ without $\left.\mathrm{C}\right)$ than the eastern one $\left(M_{\mathrm{gas}} \simeq 0.5 \times 10^{8} \mathcal{M}_{\odot}\right.$ without $\left.\mathrm{C}\right)$. The $\mathrm{CO}(1-0)$ emission cannot be associated with a single straight line but rather delineates an "S-like" shape.

The next sections will deal with the modeling of the complex gas distribution in NGC 3718 for a better understanding and a more quantitative interpretation. We will also examine in detail the effects of missing short spacings on our data by applying our procedure to a model and comparing the results thus obtained with the observations. 

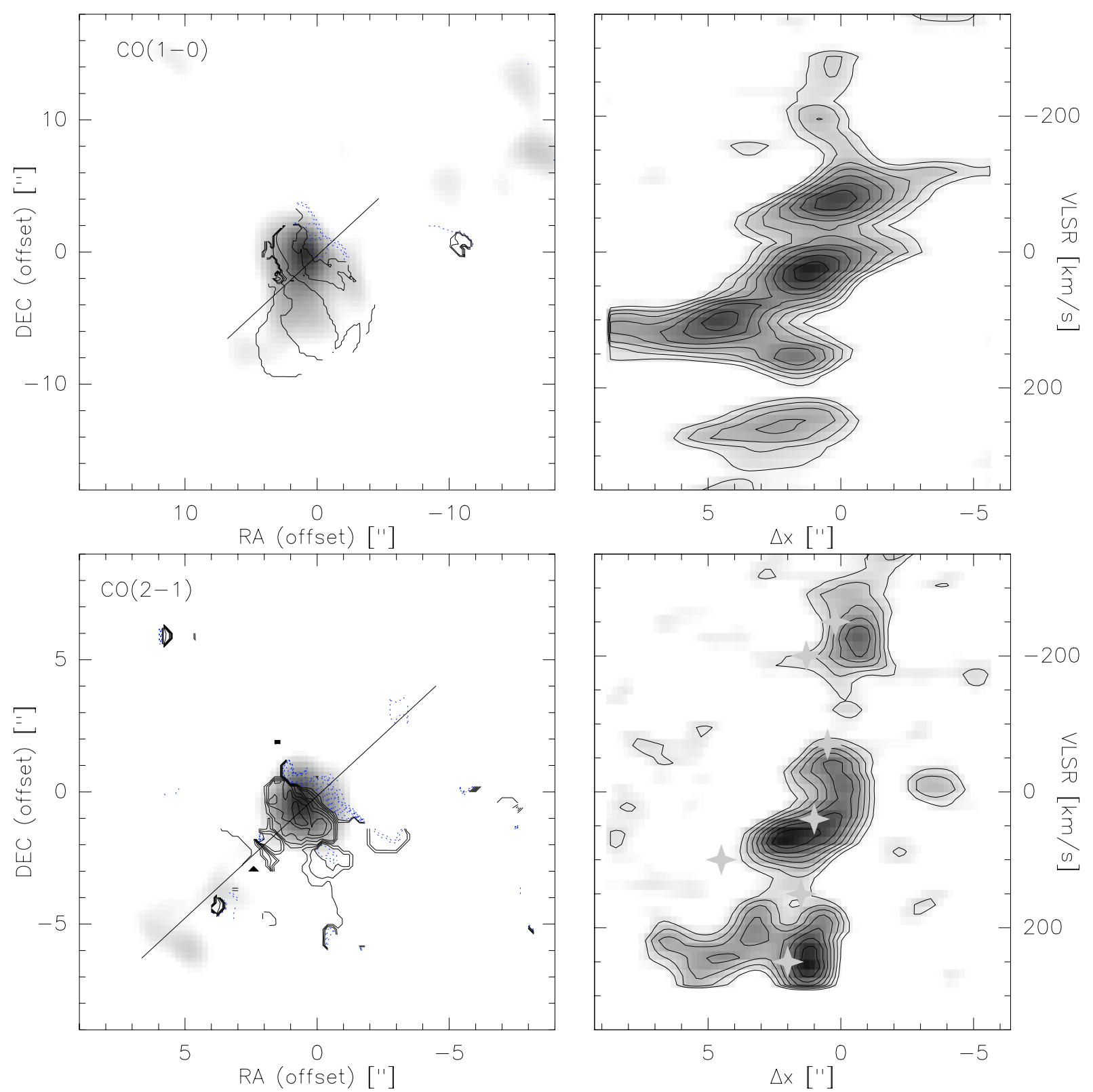

Fig. 7. Mean velocity fields of the $\mathrm{CO}(1-0)$ (upper left) and $\mathrm{CO}(2-1)$ (lower left) lines with their respective position-velocity maps $(\mathrm{CO}(1-0)$ : upper right; $\mathrm{CO}(2-1)$ lower right) taken along the axis indicated in the maps (solid line: major axis). The grey scale in the images on the right is the integrated line emission of $\mathrm{CO}(1-0)$ and $\mathrm{CO}(2-1)$ respectively. The light grey stars in the lower right panel illustrate the positions of the $\mathrm{CO}(1-0)$ components identified in the upper right panel. Left: velocity contours are in steps of $25 \mathrm{~km} \mathrm{~s}^{-1}$ for $\mathrm{CO}(1-0)$ and for $\mathrm{CO}(2-1)$. Right: contour levels are in steps of $10 \%$ from the peak starting at $30 \%$. The position velocity maps were Hanning smoothed.

\section{Modeling the $\mathrm{CO}$ distribution}

The special characteristics of the H I data for NGC 3718 led to the conclusion (Schwarz 1985) that the atomic gas is warped at radii $\gtrsim 8 \mathrm{kpc}$ in a configuration similar to that of the dust lane. IRAM $30 \mathrm{~m}$ observations of the molecular gas by Pott et al. (2004) showed this warp extending down to smaller radii of $\sim 1 \mathrm{kpc}$. Previous modelling based on the Pott et al. CO and the HI data can hence be fine-tuned with the new information gained from our significantly increased spatial resolution 4-7" for our PdBI-only map vs. 21" for the Pott et al. (2004) $30 \mathrm{~m}$ map.

\subsection{The model}

Our simulations of the mosaic maps are based on a tilted ring model similar to that of Pott et al. (2004); that paper presents more modelling details. Since the Pott et al. (2004) model of NGC 3718 reproduces the $30 \mathrm{~m}$ observations with only 3 main maxima, we had to modify the parameters to adapt their model to our PdBI results. The revised best-fit parameters were obtained by starting with the model of Pott et al. (2004), propagating it through the PdBI+30 m response function (described in Sect. 4.2), comparing the result with the observed data $(\mathrm{PdBI}+30 \mathrm{~m})$, and finally tuning the parameters until the match is optimal. In the new best-fit model, we mainly 


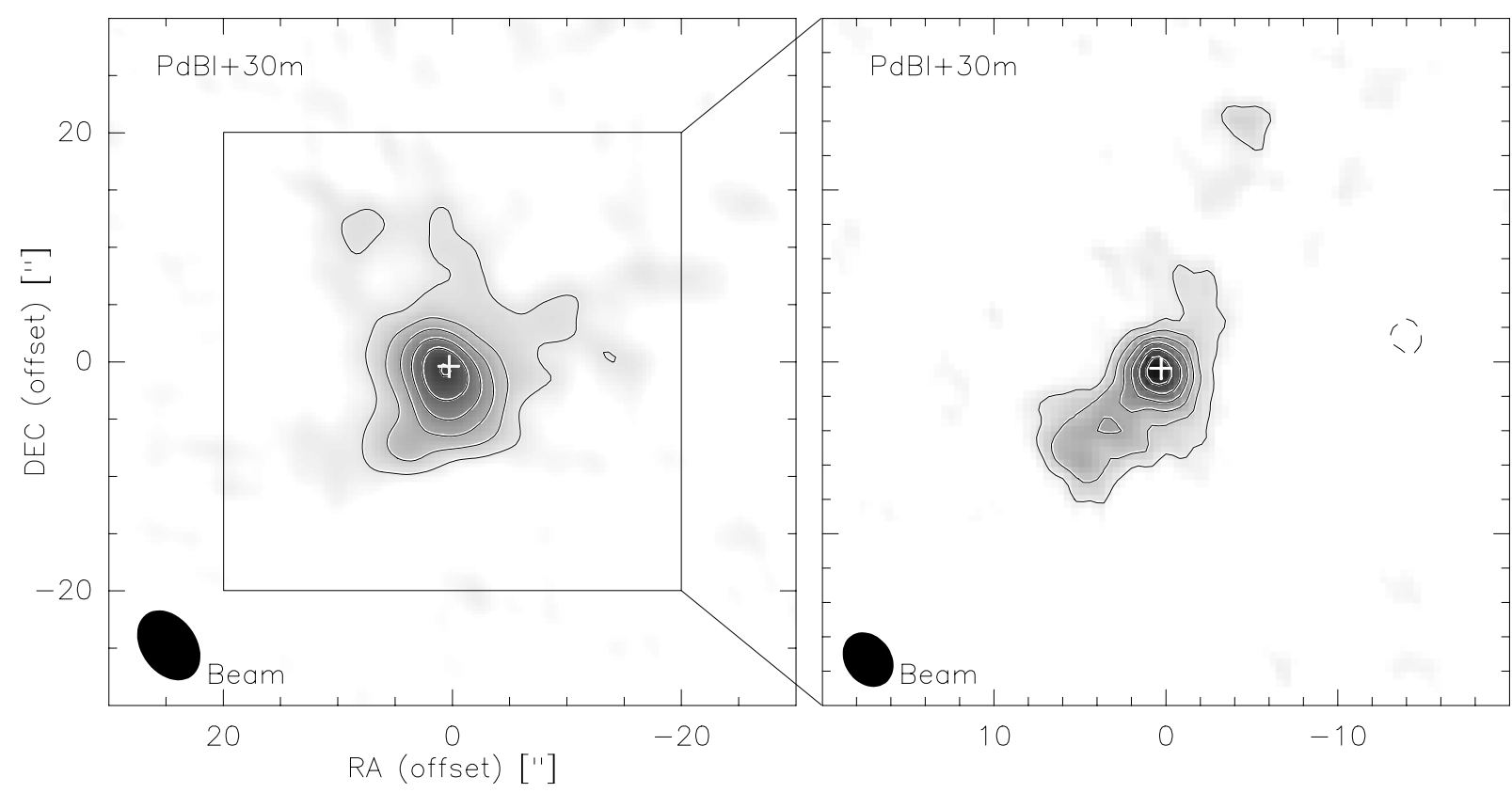

Fig. 8. PdBI central maps corrected with $30 \mathrm{~m}$ data for short-spacing effects of the integrated $\mathrm{CO}(1-0)($ left $)$ and $\mathrm{CO}(2-1)($ right $)$ emission in NGC 3718 at same size. Contour levels are from $5 \sigma(3 \sigma)=2.0(2.4)$ to $8.0(10.4) \mathrm{Jy} \mathrm{beam}^{-1} \mathrm{~km} \mathrm{~s}^{-1}$ in steps of $3 \sigma(2 \sigma)$ for $\mathrm{CO}(1-0)(\mathrm{CO}(2-1))$. The white cross indicates the position of the AGN radio continuum source at $18 \mathrm{~cm}$ derived from EVN data.

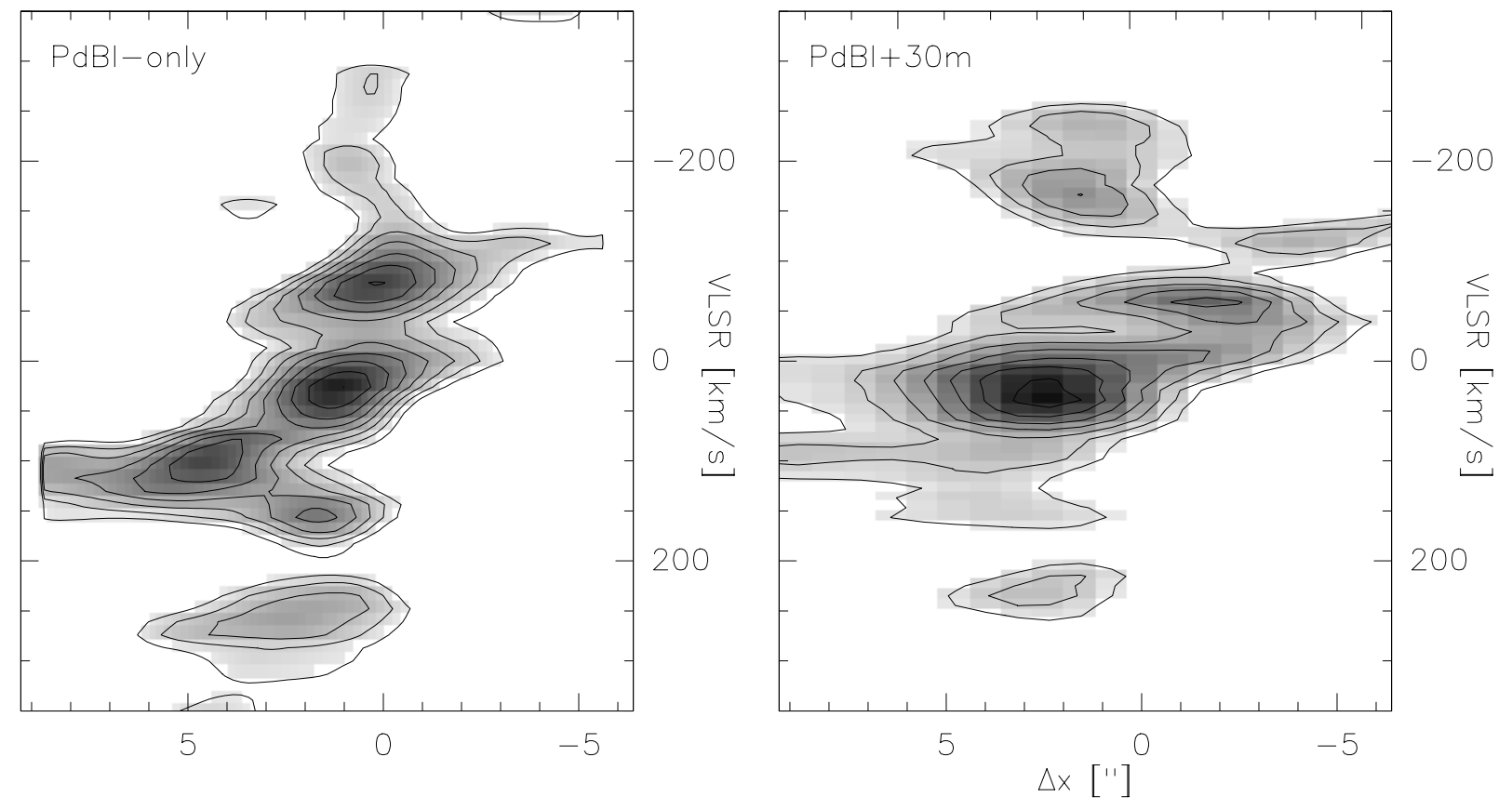

Fig. 9. Comparison of pv-diagram along axis shown in Fig. 7 between PdBI-only data for the central pointing $(l e f t)$ and PdBI $+30 \mathrm{~m}$ data for the central mosaic (right). The asymmetry is lowered in the central mosaic map but does not disappear totally. Thus, it cannot be totally due to missing short spacings. Contour levels are in steps of $10 \%$ from the peak starting at $30 \%$. The position velocity maps were Hanning smoothed.

use different tilt angles $\omega(r)$ (of a given ring relative to the assumed plane of the innermost reference disk) for the inner radii $\left(\leq 50^{\prime \prime}\right)$. Our inner rings are less warped, i.e., the tilt angles are smaller for radii from $40^{\prime \prime}$ to $60^{\prime \prime}$ than in the previous model, but they remain constant down to radii of $\sim 1^{\prime \prime}$ (i.e., in our model, the warp continues down to a radius of $\sim 1^{\prime \prime}$; see Fig. 12). Furthermore, we use a different intensity profile from Pott et al. (2004). The earlier work assumed an integrated line flux distribution for the rings that is very bright at the center but then decreases rapidly, while the intensities in our model show a slower decrease with radius. We also adopt a different inclination $\left(70^{\circ}\right.$ instead of $\left.60^{\circ}\right)$ and position angle $\left(-60^{\circ}\right.$ instead of $-85^{\circ}$ ) for the galactic disc in space. The rotation curve, however, remains the same for the new model. The integrated map of our model is shown in Fig. 13 (the model has not yet been filtered through the interferometer or single dish response 


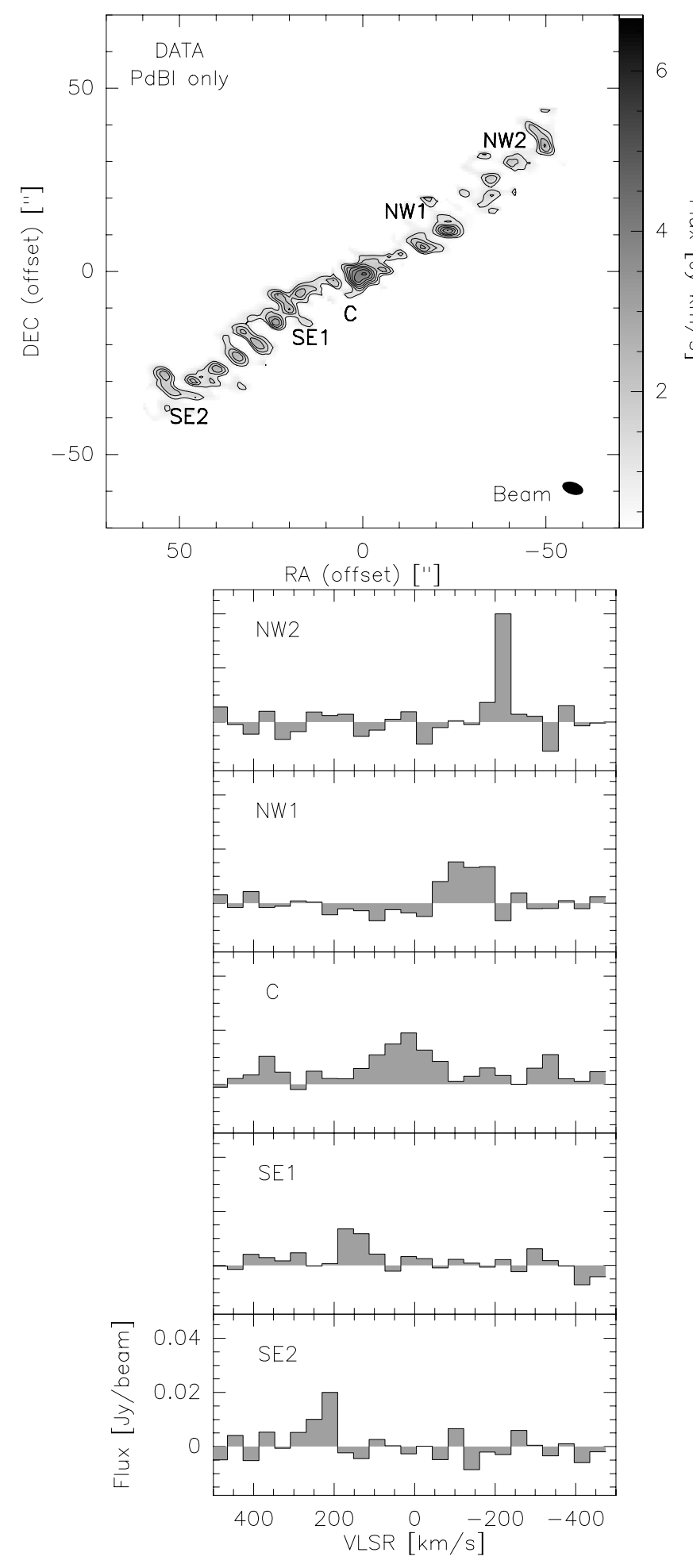

Fig. 10. PdBI mosaic map of the velocity integrated $\mathrm{CO}(1-0)$ emission in NGC 3718 (upper figure; from -300 to $+300 \mathrm{~km} \mathrm{~s}^{-1}$ ). Spectra are shown in the lower panel. Contour levels are from $2 \sigma=$ $1.0 \mathrm{Jy}_{\text {beam }}{ }^{-1} \mathrm{~km} \mathrm{~s}^{-1}$ to $4 \mathrm{Jy}_{\text {beam }}{ }^{-1} \mathrm{~km} \mathrm{~s}^{-1}$ in steps of $1 \sigma$.

function). While the central (C) and the two outer (SE2, NW2) components are quite compact and bright, the two inner features (SE1, NW1) are rather weak and relatively extended on $\sim 10^{\prime \prime}$ scales.
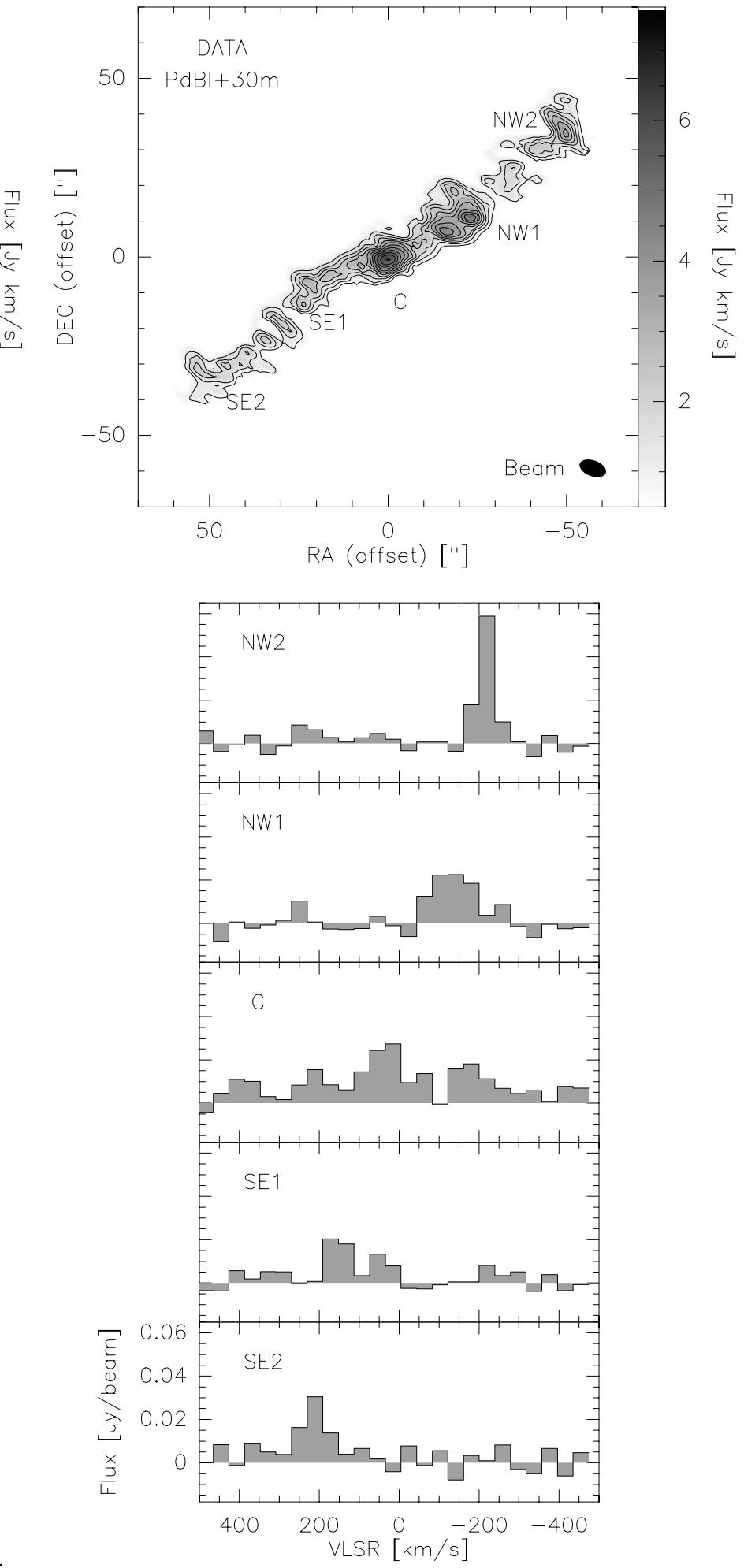

Fig. 11. Velocity integrated intensity map of the $\mathrm{CO}(1-0)$ emission for the combined PdBI $+30 \mathrm{~m}$ data (fromm -300 to $+300 \mathrm{~km} \mathrm{~s}^{-1}$ ). Contour levels are from $2 \sigma=1.0 \mathrm{Jy}$ beam ${ }^{-1} \mathrm{~km} \mathrm{~s}^{-1}$ to $6.0 \mathrm{Jy} \mathrm{beam}^{-1} \mathrm{~km} \mathrm{~s}^{-1}$ in steps of $1 \sigma$.

\subsection{Simulating the short-spacing correction}

To allow for a direct comparison between the derived model and the CO data (PdBI-only, PdBI+30 m, $30 \mathrm{~m}$-only), we simulated the effect of the different observations on the model. To 


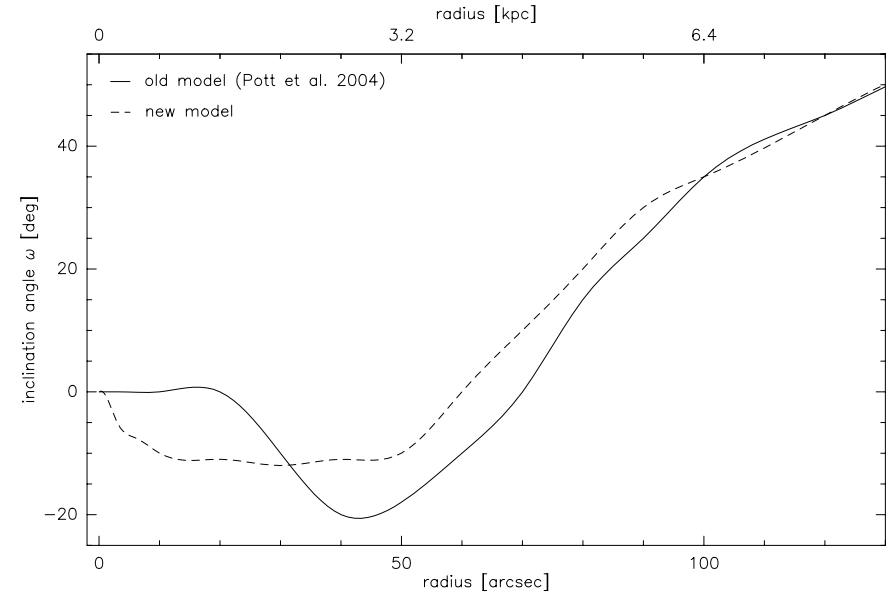

Fig. 12. Tilt angles $\omega(r)$. The old model of Pott et al. (2004) is given as a solid line, while a dashed line is used for our new model.

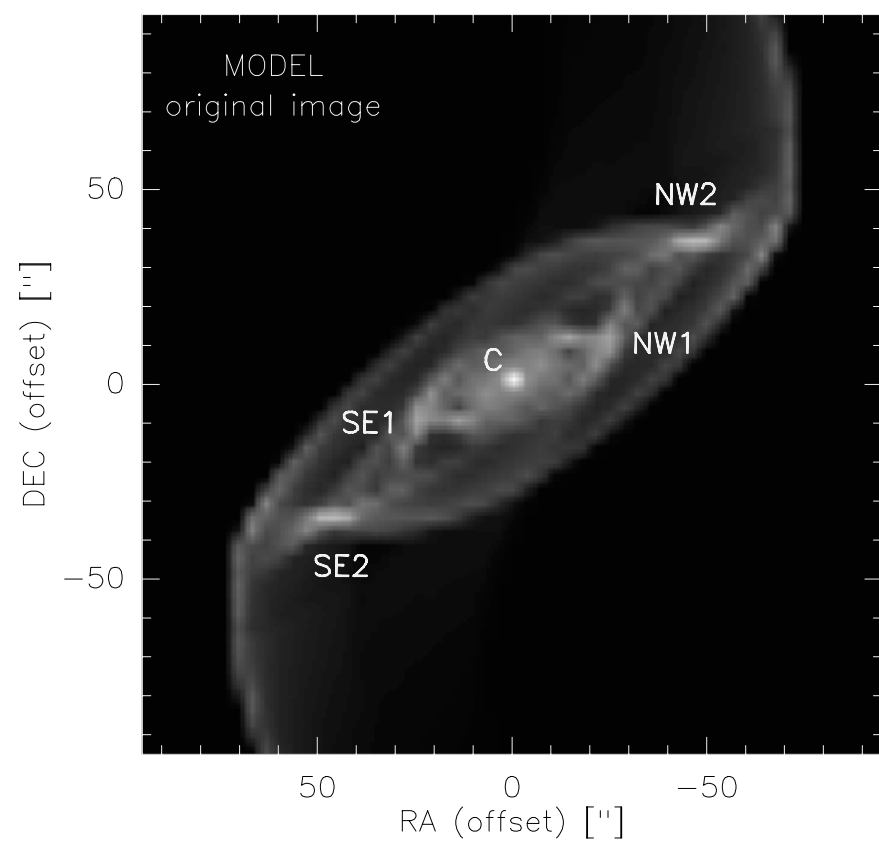

Fig. 13. The integrated intensity map of our model is plotted here, before spatial filtering to match the response of the interferometer and single dish telescope.

determine how our model galaxy would look when observed with the interferometer (before and after short-spacing correction), we first produced the simulated PdBI map with a program that computes an interferometric image (upper panel of Fig. 14) from a given $u v$ coverage (taken here from the observations) and the given three-dimensional intensity distribution (taken here from our best-fit model shown in Fig. 13). Only the most compact features are visible in the PdBI-only map while the extended emission is resolved, resulting in a loss of around $60 \%$ of the original flux. These compact features correspond to the compact orbital crowding points SE1, SE2, NW1 and NW2 (Fig. 13). The remaining emission from the tilted rings appears to be extended and mainly concentrated around SE1 and NW1. It cannot be seen with the PdBI alone due to spatial filtering, meaning that only the five compact crowding points remain in

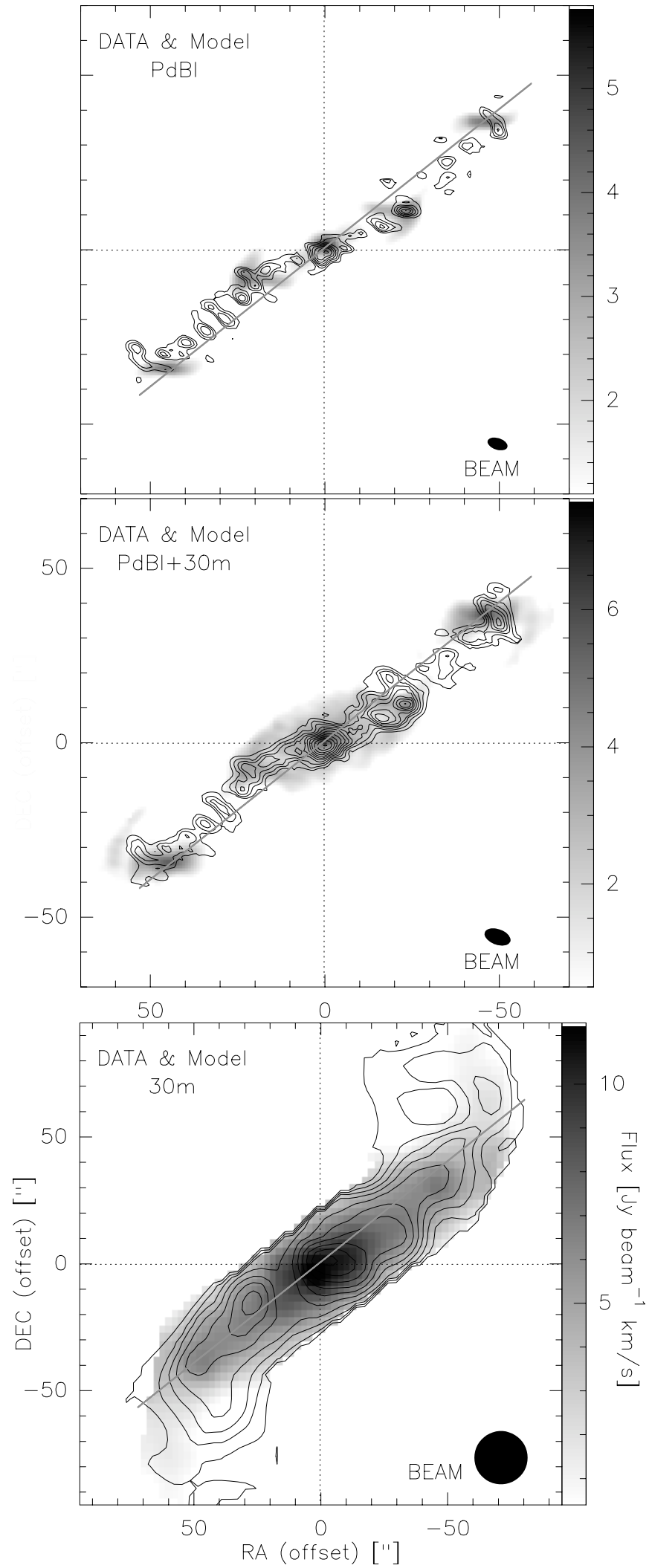

Fig. 14. Model vs. observation. Integrated maps of the PdBI data only (upper panel), the PdBI and $30 \mathrm{~m}$ data combined (middle panel) and the $30 \mathrm{~m}$ data only (lower panel). The model (grey scale) is overlaid with solid contours of the observed data. Contour levels: $P d B I$ only $2 \sigma=1.0$ to $4 \mathrm{Jy}_{\text {beam }}{ }^{-1} \mathrm{~km} \mathrm{~s}^{-1}$ by $1 \sigma ; P d B I+30 \mathrm{~m} 2 \sigma=1.0$ to $6.0 \mathrm{Jy} \mathrm{beam}^{-1} \mathrm{~km} \mathrm{~s}^{-1}$ by $1 \sigma ; 30 \mathrm{~m}$-only $3 \sigma=1.0$ to 10 by $1.0 \mathrm{Jy}_{\text {beam }}{ }^{-1} \mathrm{~km} \mathrm{~s}^{-1}$. 
the PdBI-only map. The extended emission around SE1 and NW1 is thus heavily resolved (compare Fig. 13). The bestfit model was also smoothed to the beam $\left(\sim 21^{\prime \prime}\right)$ of the $30 \mathrm{~m}$ telescope and the spectra were taken at the same positions as for the observations. Accounting for normalisation, these data were transformed into the $30 \mathrm{~m}$ file that would have been produced if such a distribution had been observed with the IRAM $30 \mathrm{~m}$ telescope. This file is thus suitable for comparison with the measured $30 \mathrm{~m}$ data (see lower panel in Fig. 14). We indeed find only three main maxima, a merging of the inner two (SE1, NW1) with the outer two maxima (SE2, NW2) and an S-line morphology.

As a final test, we combined the simulated $30 \mathrm{~m}$ and PdBI maps. All three simulated maps are plotted in Fig. 14 (grey scale). The loss of flux due to the reduced sensitivity to diffuse extended emission in the interferometer-only data is indeed reproduced by the model. A comparison of the simulated peak fluxes from the PdBI, the PdBI+30 m, and the $30 \mathrm{~m}$ data with each other suggests that the PdBI has lost a factor $\sim 2-3$ of the peak line flux, consistent with what is observed. The shortspacing correction not only reproduces the correct fluxes but also the correct distribution without any artefact or inconsistency in excess of $1 \%$ for the model.

\subsection{Model vs. data}

We have carried out the same steps to combine the observed PdBI and $30 \mathrm{~m}$ data (Sect. 3.4). The results are plotted in Fig. 14 as contours over the simulated maps. We find a very similar behavior in the observed and model maps. Besides the inner two maxima which are more easily visible, the flux level also increases. Thus, a better distinction between signal and noise is possible. As the two eastern components are less dominant (by a factor 2) than the western components in the integrated map over the whole velocity range, we show separate plots for each component integrated over the appropriate velocity range (see Fig. 15). Such an asymmetry of the gas distribution has already been found in many other galaxies as well (e.g., Richter \& Sancisi 1994) and cannot be explained by our symmetric tilted ring model. It might be an indication for a tidal interaction with the companion galaxy which would result in an asymmetric gas distribution. Thus, our warp model could presumably be improved by adding asymmetries to account for the putative interaction.

The maps of the data (left side) and the model (right side) are separately shown in Fig. 16, along with the good agreement between the model and the data in terms of spectra taken at each of the five components. A somewhat weaker eastern component is also visible in the model. This is due to the asymmetric sampling of the $30 \mathrm{~m}$-spectra with respect to the CO centroid. Simulated and observed line widths are consistent within the noise. In Fig. 17, we have derived first order moment maps with a $\sim 3 \sigma$ clipping over a velocity range of $\sim-300 \mathrm{~km} \mathrm{~s}^{-1}$ to $+300 \mathrm{~km} \mathrm{~s}^{-1}$ and taken position-velocity cuts along the major axis of the galaxy for the observed and simulated data. Compared to the pv-diagram of a standard gas disk, a more complex structure is seen in the pv-diagrams of the observations as well as of the simulation due to orbital crowding effects. As might be expected for a rapidly rotating nuclear disk with a size of $\sim 1.5 \mathrm{kpc}$, a steep linear rise is indicated in the position-velocity diagram. This feature agrees well in orientation and size with the central field velocity component visible in the $\mathrm{CO}(1-0)$ map of Fig. 7. We again find that this central feature in the position-velocity map extends only over a range of $\pm 10^{\prime \prime}$ in Fig. 17, which is smaller than the interval derived by Pott et al. (2004). The difference from the position velocity diagram in Pott et al. (2004) has a logical and simple explanation. In their maps, $\{\mathrm{SE} 1, \mathrm{SE} 2\}$ and $\{\mathrm{NW} 1, \mathrm{NW} 2\}$ merge (in the $30 \mathrm{~m}$ beam) into almost single SE and NW features, respectively, and thus all lie on the major axis. In our interferometric map, SE1 and NW1 are no longer aligned with this major axis and thus appear only weakly in the position-velocity diagram of Fig. 17. The high velocity gas returns if the major axis is rotated by about $\sim 10^{\circ}$. We thus have three different velocity regimes. The first ranges from $\pm 10^{\prime \prime}$ with $\Delta v \sim \pm \sim 150 \mathrm{~km} \mathrm{~s}^{-1}$ and can be identified with the gas occupying the inner rings. The second region extends to $\pm 30^{\prime \prime}$ with $\Delta v \sim \pm 220 \mathrm{~km} \mathrm{~s}^{-1}$ and is caused by intermediate rings. The third extends to $\pm 70^{\prime \prime}$ with $\Delta v= \pm 220 \mathrm{~km} \mathrm{~s}^{-1}$ and originates from the outer rings.

\section{Discussion and conclusions}

The paper presents an analysis based on arcsec angular resolution PdBI observations of the gas distribution in NGC 3718, complementing previous studies conducted by Pott et al. (2004: CO on $\geq 20^{\prime \prime}$ scales) and Schwarz (1985: HI on $\geq 30$ " scales). After making a short-spacing correction using the IRAM $30 \mathrm{~m}$ data (Pott et al. 2004), we have modelled and interpreted the mosaic observations of the total emission as well as single field observations of the central kpc. Three compact and three extended components were found in the mosaic map of the $\mathrm{CO}(1-0)$ emission. At the higher angular resolution of the PdBI, the gas structure turns out to be more complex than previously appreciated. To explain the detected molecular gas distribution, we were forced to modify the Pott et al. tilted ring model slightly. The revised model now not only reproduces well the PdBI+30 m observations, but due to the higher resolution of the observations, also places much tighter constraints on the warp. The disk is most likely already warped on arcsec scales $\left(\sim 4^{\prime \prime} \equiv 250 \mathrm{pc}\right)$ and is evident within a radius of $20^{\prime \prime}$ (the S-like shape between SE1, C, and NW1) even if the warp is less dominant within $50^{\prime \prime}$ relative to the original Pott et al. model. The five symmetric CO(1-0) peaks (SE2, SE1, C, NW1, and NW2) detected by the PdBI can be explained by orbit crowding effects. The two outer maxima merge into one feature on each side of the center in the beam of the IRAM $30 \mathrm{~m}$ telescope and thus at this resolution appear as single components. Besides the gas distribution, the gas kinematics can be almost completely explained by the model. The position-velocity diagrams of both data sets - mosaic and central pointing - unveil rapidly rotating nuclear gas within a radius of $\sim 700 \mathrm{pc}$, as seen in the simulated data. The high velocities in the center are produced by the inner warped rings. Thus, the dynamics of the molecular gas can be traced back to a continuous warped disk with concentric orbits. 


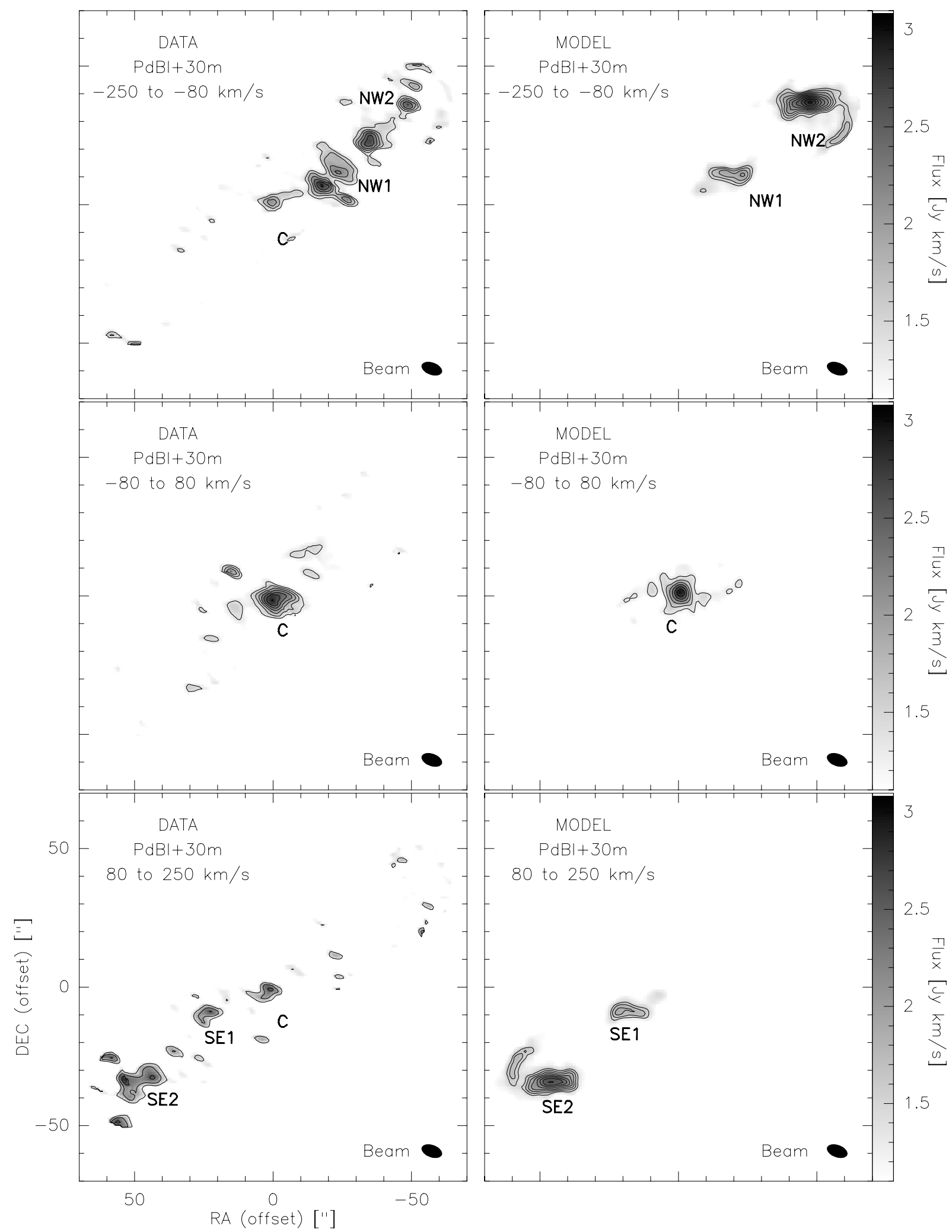

Fig. 15. Model vs. Observation. Integrated maps of the measured (left) and simulated (right) PdBI+30 m data restricted to the following velocity ranges: -250 to $-80 \mathrm{~km} \mathrm{~s}^{-1}$ (upper panel), -80 to $80 \mathrm{~km} \mathrm{~s}^{-1}$ (middle panel), and 80 to $225 \mathrm{~km} \mathrm{~s}^{-1}$ (lower panel). Contour levels are $3 \sigma=1.2 \mathrm{Jy}$ beam $^{-1} \mathrm{~km} \mathrm{~s}^{-1}$ in steps of $1 \sigma$.

As in Pott et al. (2004), our model does not require a bar to explain the observed gas properties down to $\sim 250 \mathrm{pc}$. However a warp alone is not sufficient to account for all data, it must be combined with asymmetry in gas density between the eastern and western side. This asymmetry is logically coming from the same mechanism producing the warp: i.e. an interaction with 
M. Krips et al.: Molecular gas in NUclei of GAlaxies (NUGA). III.
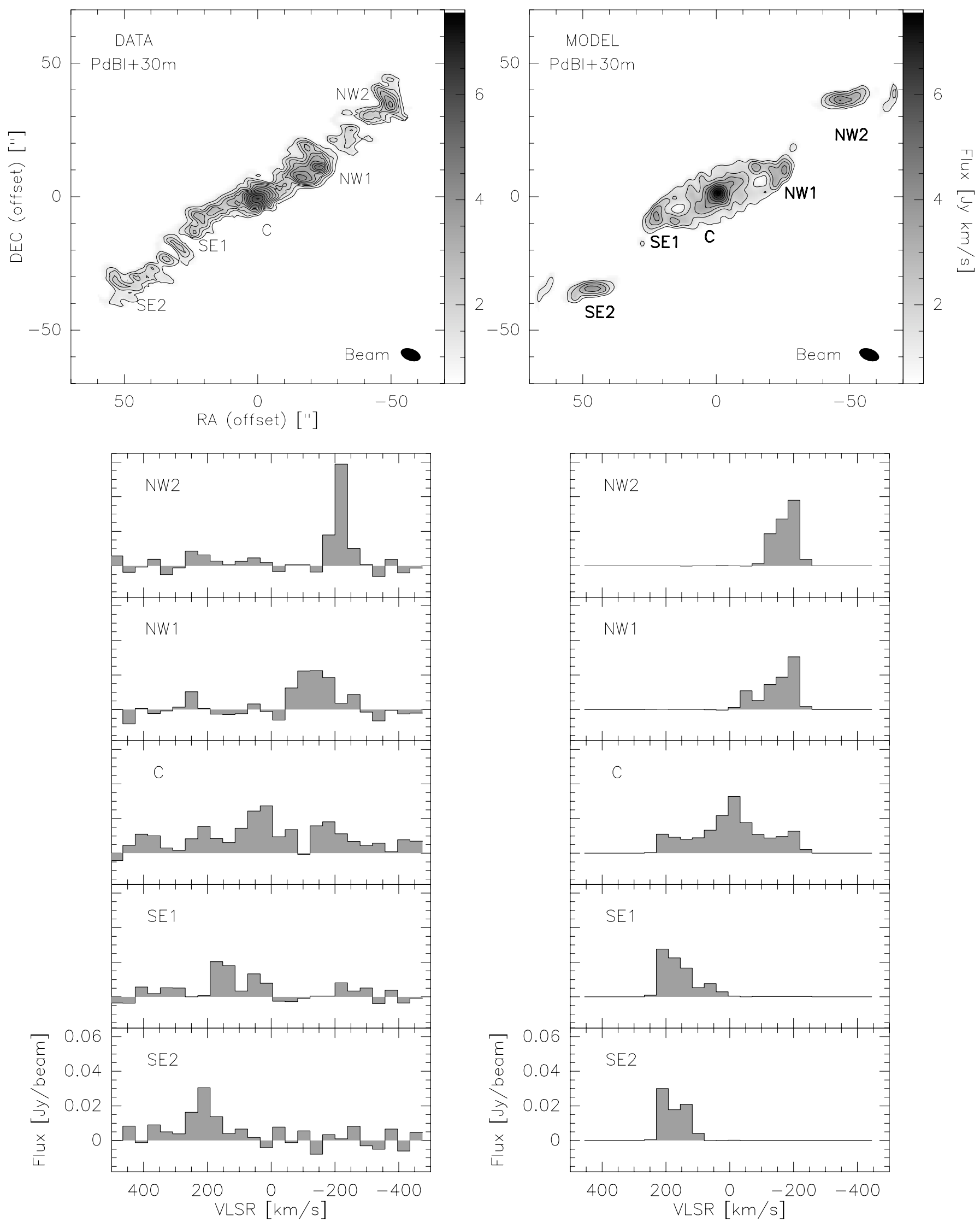

Fig. 16. Upper panels: the short-spacing corrected images of the observed data (left) and of the model (right) data. Lower panels: $30 \mathrm{~m}$ spectra at positions of the CO peaks. Contour levels are from $2 \sigma=1.0$ to $6.0 \mathrm{Jy} \mathrm{beam}^{-1} \mathrm{~km} \mathrm{~s}^{-1}$ in steps of $1 \sigma$. 

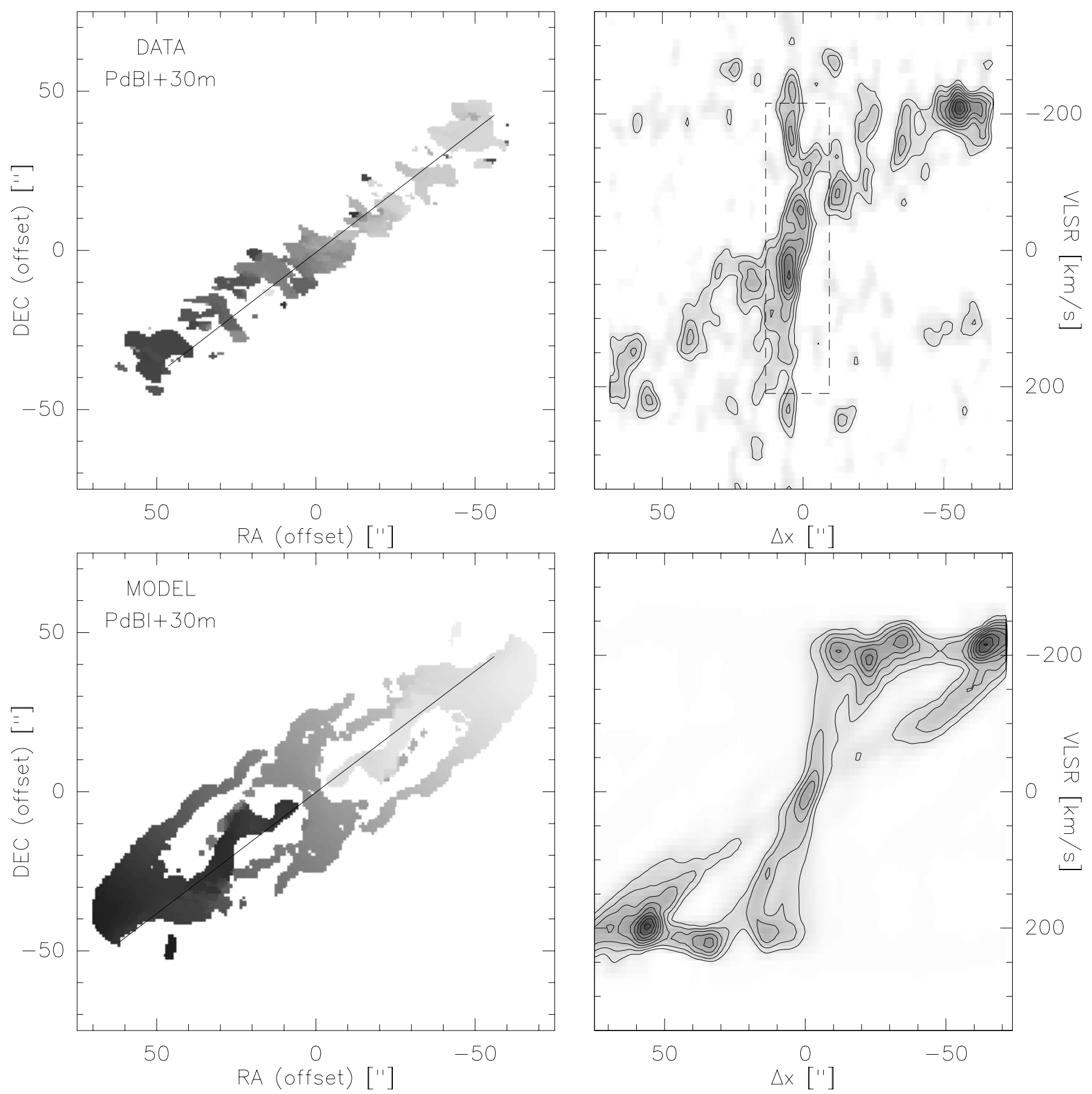

Fig. 17. Upper panels: observed data, with first order moment maps (left) and position velocity cuts (right) taken along the axis indicated in the first order moment map (going through the AGN). Lower panels: same as above for the model. The area within the dashed box is identical with the map shown in the right panel of Fig. 9. Contour levels are in steps of $10 \%$ from the peak starting at $20 \%$. The position velocity maps were Hanning smoothed.

the companion galaxy. The asymmetric gas distribution within the central $250 \mathrm{pc}$ provides the possibility for an accretion of gas onto the central engine. However, the transport of gas to the black hole still raises many questions and problems. Even in the Milky Way, where a warped circumnuclear molecular torus (Guesten et al. 1987) provides a reservoir for accretion at a distance of only $\sim 1 \mathrm{pc}$ from the nucleus, the situation is not yet understood. In this context, the mechanisms of gas fueling to the inner few pc/subpc in active nuclei appear even more complex. Besides the comprehension of our own Galactic center, only detailed studies of further nuclei with the highest angular resolution can provide answers to the wealth of open questions.

Acknowledgements. The research presented in this paper has been financially supported in part by the SFB 494. Stephane Leon is partially supported by DGI Grant AYA 2002-03338 and Junta de Andalucía. We thank the IRAM staff from the Plateau de Bure and Grenoble for conducting the observations and helping with the data reduction. We are grateful to the referee Dr. Michel Guelin for his useful comments. 


\section{References}

Barth, A. J., Ho, L. C., Filippenko, A. V., \& Sargent, W. L. W. 1998 ApJ, 496, 133

Becker, R. H., White, R. L., \& Helfand, D. J. 1995, ApJ, 450, 559

Burke, B. F., \& Miley, G. K. 1973, A\&A, 28, 379

Combes, F. 1988, in Galactic and Extragalactic Star Formation, ed. Pdritz, \& Fich (Dordrecht: Kluwer), 475

Combes, F., García-Burillo, S., Boone, F., et al. 2004, A\&A, 414, 857

Downes, D., Solomon, P. M., \& Radford, S. J. E. 1993, ApJ, 414, L13

Duschl, W. J., Strittmatter, P. A., \& Biermann, P.L. 2000, A\&A, 357, 1123

Falcke, H. 1996, ApJ, 464, L67

Falcke, H., \& Markoff, S. 2000, A\&A, 362, 113

Falcke, H., Lehar, J., Barvainis, R., Nagar, N. M., \& Wilson, A. S. 2001, in Probing the Physics of Active Galactic Nuclei by Multiwavelength Monitoring, ed. B. M. Peterson, R. S. Polidan, \& R. W. Pogge (San Francisco: ASP), ASP Conf. Ser., 224, 265

Filippenko, A. V., \& Sargent, W. L. W. 1985, ApJS, 57, 503

García-Burillo, S., Sempere, M. J., Combes, F., Hunt, L. K., \& Neri, R. 2000 A\&A, 363, 869

García-Burillo, S., Combes, F., Eckart, A., et al. 2003a, in Active Galactic Nuclei: From Central Engine to Host Galaxy, ed. S. Collin, F. Combes, \& I. Shlohsman, ASP Conf. Ser., 290, 423

García-Burillo, S., Combes, F., Hunt, L. K., et al. 2003b, A\&A, 407, 485

Guesten, R., Genzel, R., Wright, M. C. H., et al. 1987, ApJ, 318, 124

Guilloteau, S., Delannoy, J., Downes, D., et al. 1992, A\&A, 262, 624

Guilloteay, S., \& Lucas, R. 2000, in Imaging at Radio Through Submillimeter Wavelengths, ed. J. G. Mangum, \& S. J. E. Radford (San Francisco: ASP), 299
Hartwich, M., Eckart, A., \& Leon, S. 2002, AGM, 18, 136

Heckmann, T. M., Smith, E. P., Baum, S. A., et al. 1986, ApJ, 311, 526

Keene, J., Stern, D., Lawrence, C., et al. 2004, A\&A\&S, 204, 4122 http://www.spitzer.caltech.edu/Media/releases/ ssc2004-09/release.shtml

Kormendy, J., \& Bender, R. 1999, ApJ, 522, 772

Krips, M., Pott, J.-U., Eckart, A., et al. 2003, Ap\&SS, 284, 511

Martini, P., \& Pogge, R. W. 1999, AJ, 118, 2646

Nagar, N. M., Falcke, H., Wilson, A. S., \& Ho, L. C. 2000, ApJ, 542, 186

Nagar, N. M., Wilson, A. S., \& Falcke, H. 2001, ApJ, 559, L87

Nagar, N. M., Falcke, H., Wilson, A. S., \& Ulvestad, J. S. 2002, A\&A, 392, 53

Narayan, R., Mahadevan, R., \& Quataert, E. 1998, in Theory of Blach Hole Accretion Disks, ed. M. A. Abramowicz, G. Björnsson \& J. E. Pringle (Cambridge: Cambridge Univ. press.), 148

Pott, J.-U., Hartwich, M., Eckart, A., et al. 2004, A\&A, 415, 27

Quataert, E. 2001, in Probing the Physics of Active Galactic Nuclei by Multiwavelength Monitoring, ed. B. M. Peterson, R. S., Polidan, $\&$ R. W. Pogge (San Francisco: ASP), ASP Conf. Ser., 224, 71

Richter, O.-G., \& Sancisi, R. 1994, A\&A, 290, L9

Schinnerer, E., Eckart, A., \& Tacconi, L. J. 2000a, ApJ, 533, 826

Schinnerer, E., Eckart, A., Tacconi, L. J., Genzel, R., \& Downes, D. 2000b, ApJ, 533, 850

Schwarz, U. J. 1985, A\&A, 142, 273

Shlosman, I., Frank, J., \& Begelman, M.C. 1989, Nature, 338, 45

Slee, O. B., Sadler, E. M., Reynolds, J. E., \& Ekers, R.D. 1994, MNRAS, 269, 928

Wild, W., \& Eckart, A. 2000, A\&A, 359, 483

Wrobel, J. M., \& Heeschen, D. 1991, AJ, 101, 148 